\title{
Synthesis, Photophysical Properties and Metal-Ion Recognition Studies of Fluoroionophores Based on 1-(2-Pyridyl)-4-Styrylpyrazoles
}

\author{
Jessica Orrego-Hernández, ${ }^{\dagger} J_{\text {Justo }}$ Cobo $^{\ddagger}$ and Jaime Portilla*, ${ }^{\dagger}$ \\ ${ }^{\dagger}$ Bioorganic Compounds Research Group, Department of Chemistry, Universidad de los Andes, \\ Carrera 1 No. 18A-10, 111711 Bogotá, Colombia \\ ĐDepartamento de Química Orgánica e Inorgánica, Universidad de Jaén, 23071 Jaén, Spain
}

*Email: jportill@uniandes.edu.co

\section{SUPPORTING INFORMATION}

\section{Content:}

1. Overview of Substrates, Intermediates and Products Numbering ............................. S2

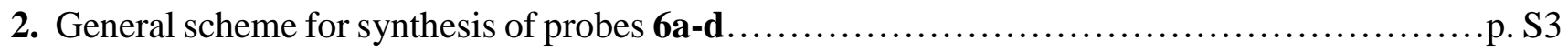

3. Supplementary Analytical Data (Identification of intermediate and byproducts).................. S3

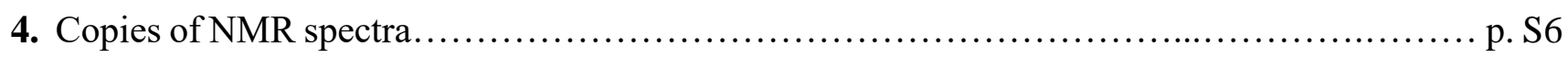

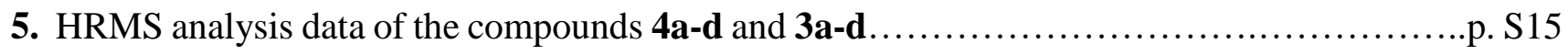

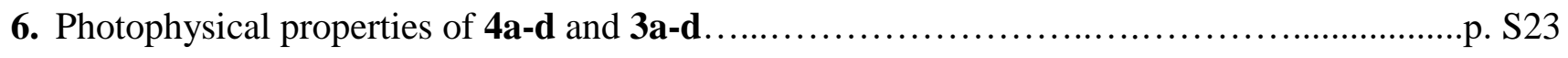

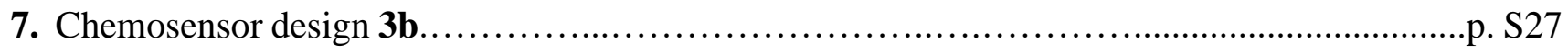




\section{Overview of Substrates Intermediates and Products Numbering}

Acetophenones 1a-c, arylhydrazines 2a-b, phosphonium salts 5a-b, and phenyl halides $\mathbf{8 a - b}$ (reactants)<smiles>CC(=O)c1ccc([N+](=O)[O-])cc1</smiles>

7a $\mathrm{Ph}_{\oplus}^{\mathrm{Ph}} \stackrel{\mathrm{Ph}}{-\mathrm{Ph} B \mathrm{Br}^{\ominus}}$

$2 a$<smiles>CC(=O)c1ccccc1</smiles>

$7 \mathrm{~b}$<smiles>COc1ccc(C(C)=O)cc1</smiles>

7c<smiles>NNc1ccccc1</smiles>

$8 a$<smiles>NNc1ccccn1</smiles>

$8 b$

Hydrazones 3a-d and 4-formylpyrazoles 4a-d (intermediates)<smiles>C/C=C\[N+](=O)[O-]</smiles><smiles>C/C(=N\Nc1ccccn1)c1ccc(C)cc1</smiles>

9a<smiles>CC[N+](=O)[O-]</smiles><smiles>O=Cc1cn(-c2ccccn2)nc1-c1ccc(F)cc1</smiles><smiles>C/C(=N\Nc1ccccn1)c1ccccc1</smiles>

$9 b$<smiles>O=Cc1cn(-c2ccccn2)nc1-c1ccccc1</smiles><smiles>Brc1ccccc1</smiles>

$5 a$<smiles>Ic1ccccc1</smiles>

5b<smiles>CCOc1ccc(/C(C)=N/Nc2ccccn2)cc1</smiles><smiles>COc1ccc(-c2nn(-c3ccccn3)cc2C=O)cc1</smiles><smiles>C/C(=N\Nc1ccccc1)c1ccccc1</smiles>

9d<smiles>O=Cc1cn(-c2ccccc2)nc1-c1ccccc1</smiles>

4-Vinylpyrazoles 7a-d and 4-styrilpyrazoles 6a-d (products)
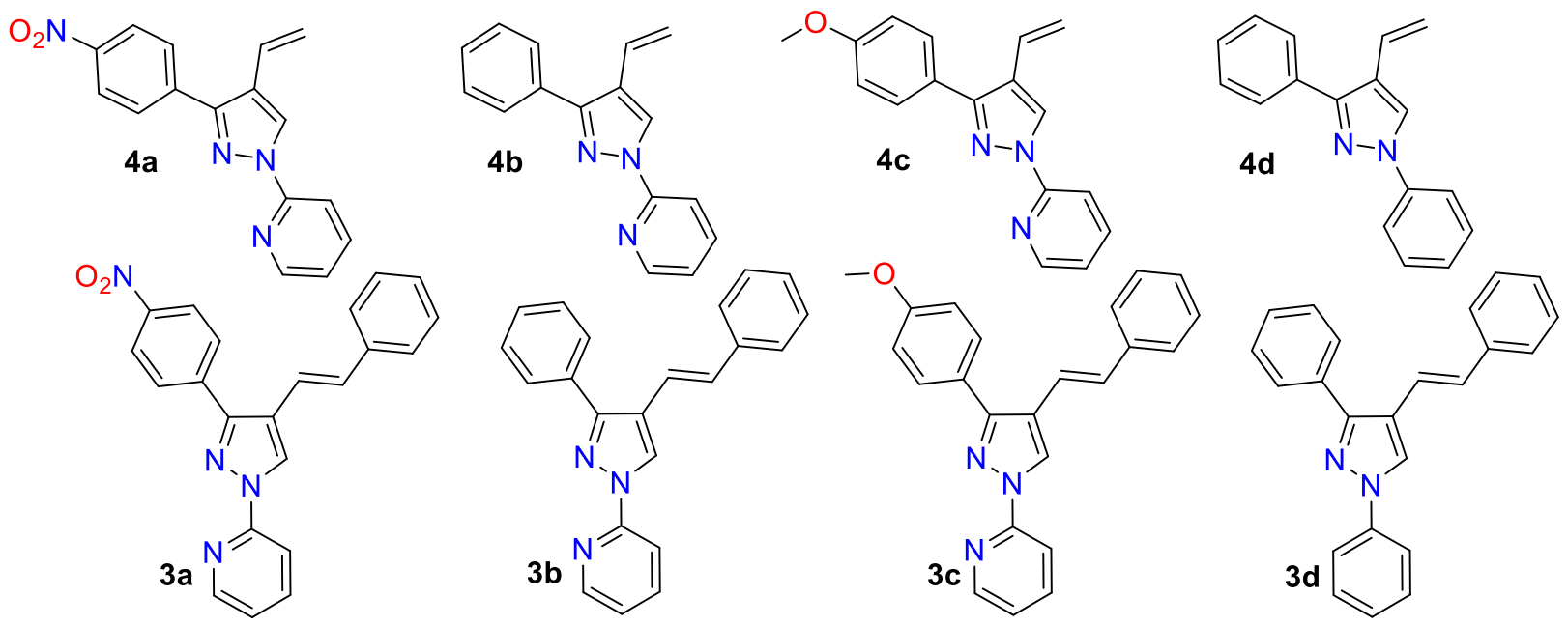

Scheme S2. Structure of all substrates intermediates and products involved in this research 


\section{General scheme for synthesis of ligands 6a-d}

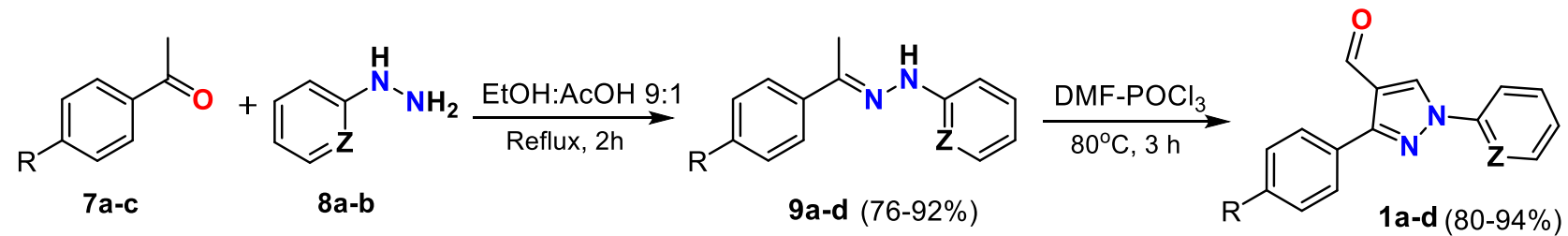

R: a) $\mathrm{NO}_{2}$, b) $\mathrm{H}$, c) $\mathrm{MeO} \quad$ Z: a) $\mathrm{CH}$, b) $\mathrm{N}$

$\mathrm{CH}_{3} \mathrm{PPh}_{3} \mathrm{Br} \mid \mathbf{2 b}$

$n$-BuLi, THF

$-40-20^{\circ} \mathrm{C}, 5 \mathrm{~h}$
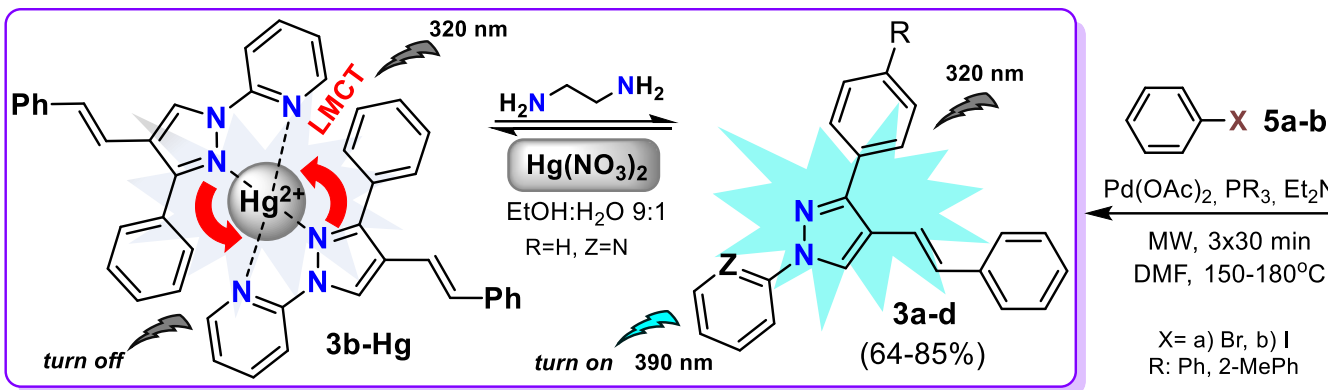

$\mathrm{Pd}(\mathrm{OAc})_{2}, \mathrm{PR}_{3}, \mathrm{Et}_{2} \mathrm{~N}$

MW, $3 \times 30 \mathrm{~min}$

DMF, $150-180^{\circ} \mathrm{C}$

$\mathrm{X}=\mathrm{a}) \mathrm{Br}, \mathrm{b}) \mathrm{I}$

$\mathrm{R}: \mathrm{Ph}, 2-\mathrm{MePh}$

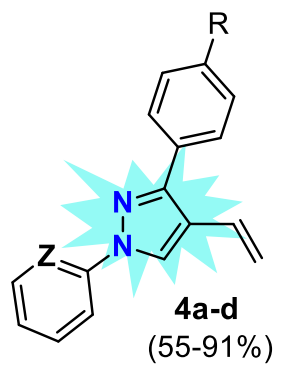

Scheme S2. General synthetic route for the formation of ligands 3a-d starting from acetophenones

\section{Supplementary Analytical Data (Identification of intermediate and byproducts)}

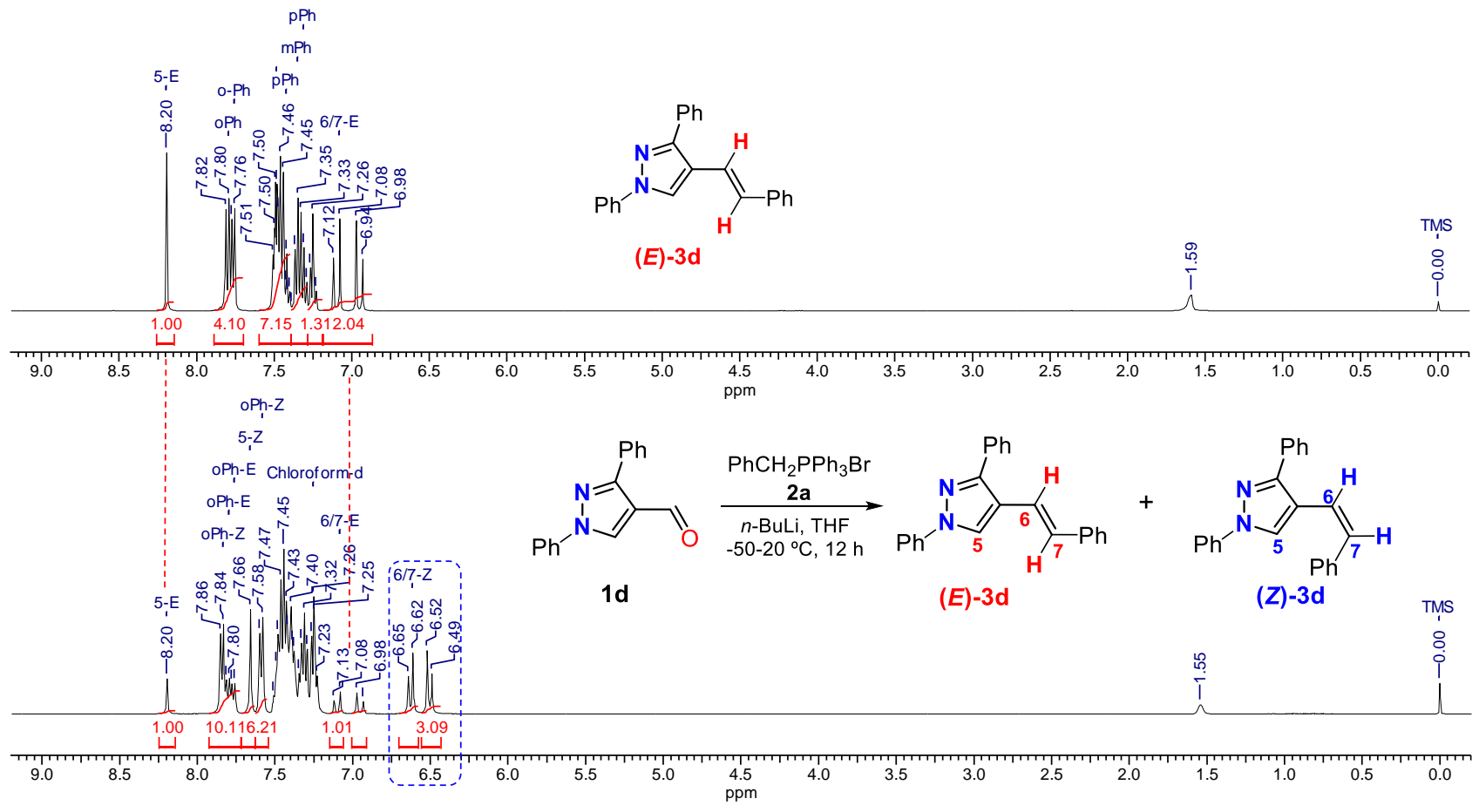

Figure S1. Identification by ${ }^{1} \mathrm{H}$ NMR $\left(\mathrm{CDCl}_{3}\right)$ of the 4-styrylpyrazoles $(\boldsymbol{Z})$-3d and $(\boldsymbol{E})-\mathbf{3 d}$. 


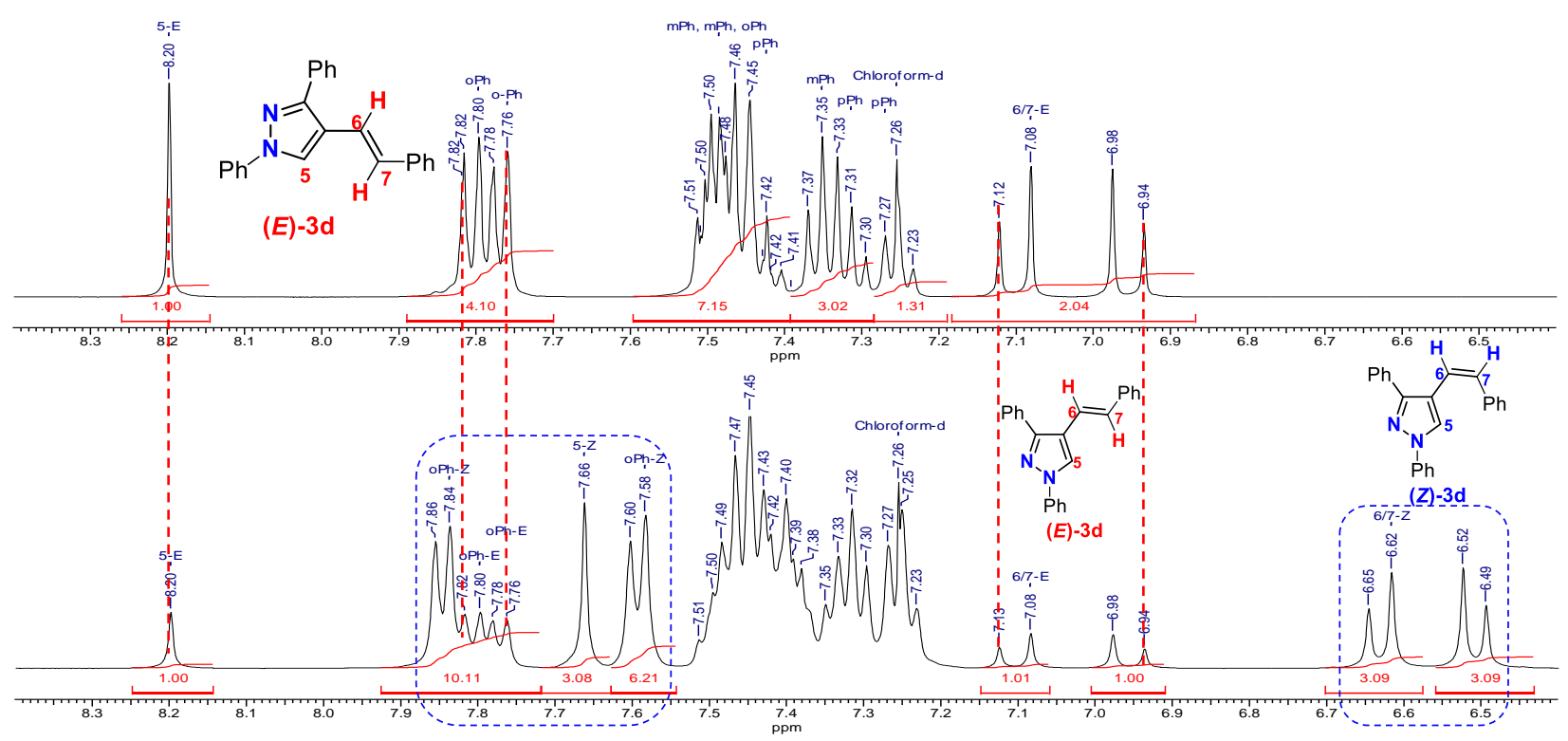

Figure S2. Expansion ${ }^{1} \mathrm{H}$ NMR spectra for the 4-styrylpyrazoles (Z)-3d and (E)-3d.
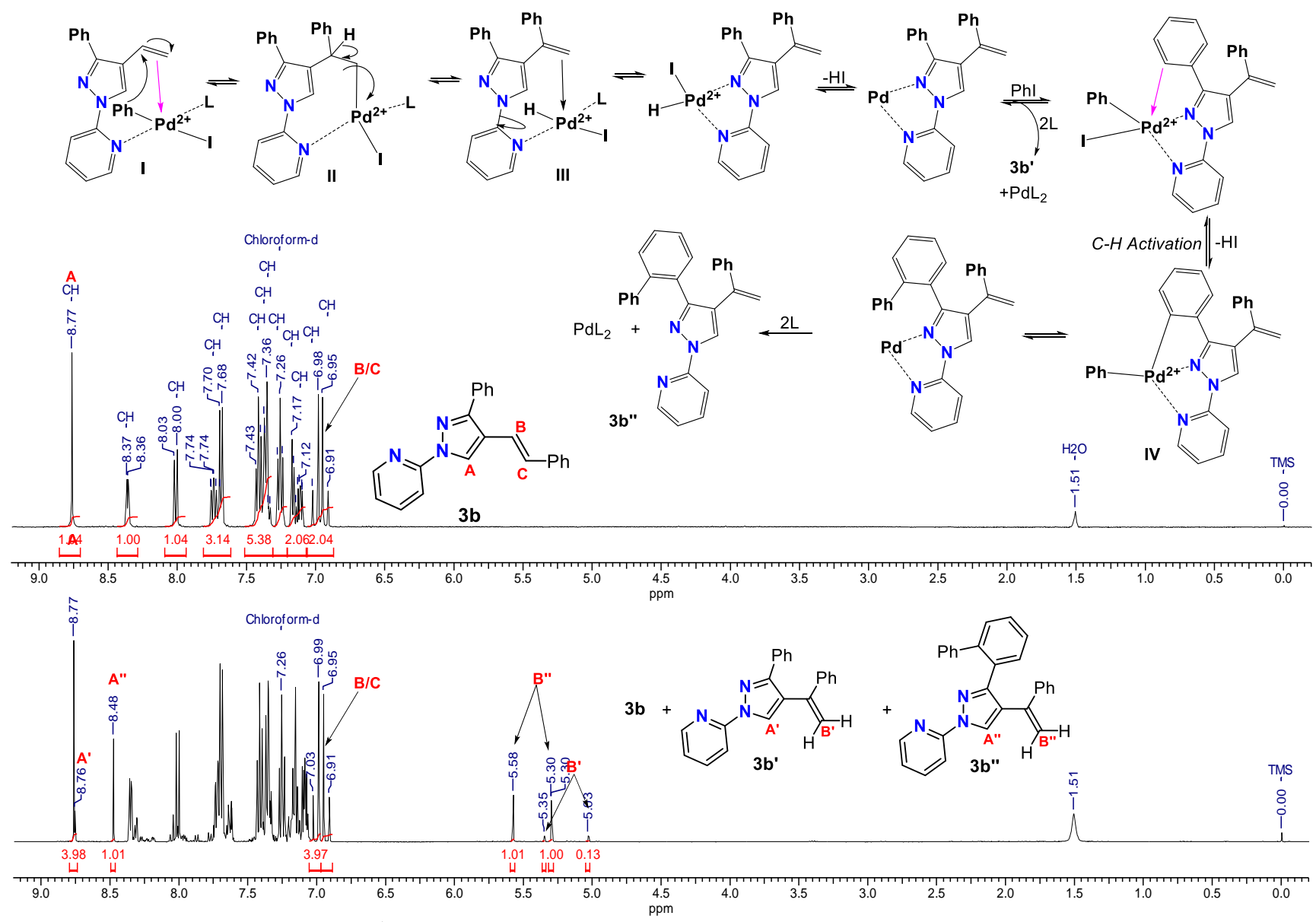

Figure S3. Identification by ${ }^{1} \mathrm{H}$ NMR $\left(\mathrm{CDCl}_{3}\right)$ of $\mathbf{3 b}, \mathbf{3} \mathbf{b}^{\prime}$ and $\mathbf{3} \mathbf{b}^{\prime \prime}$. Plausible mechanism for this reaction. 


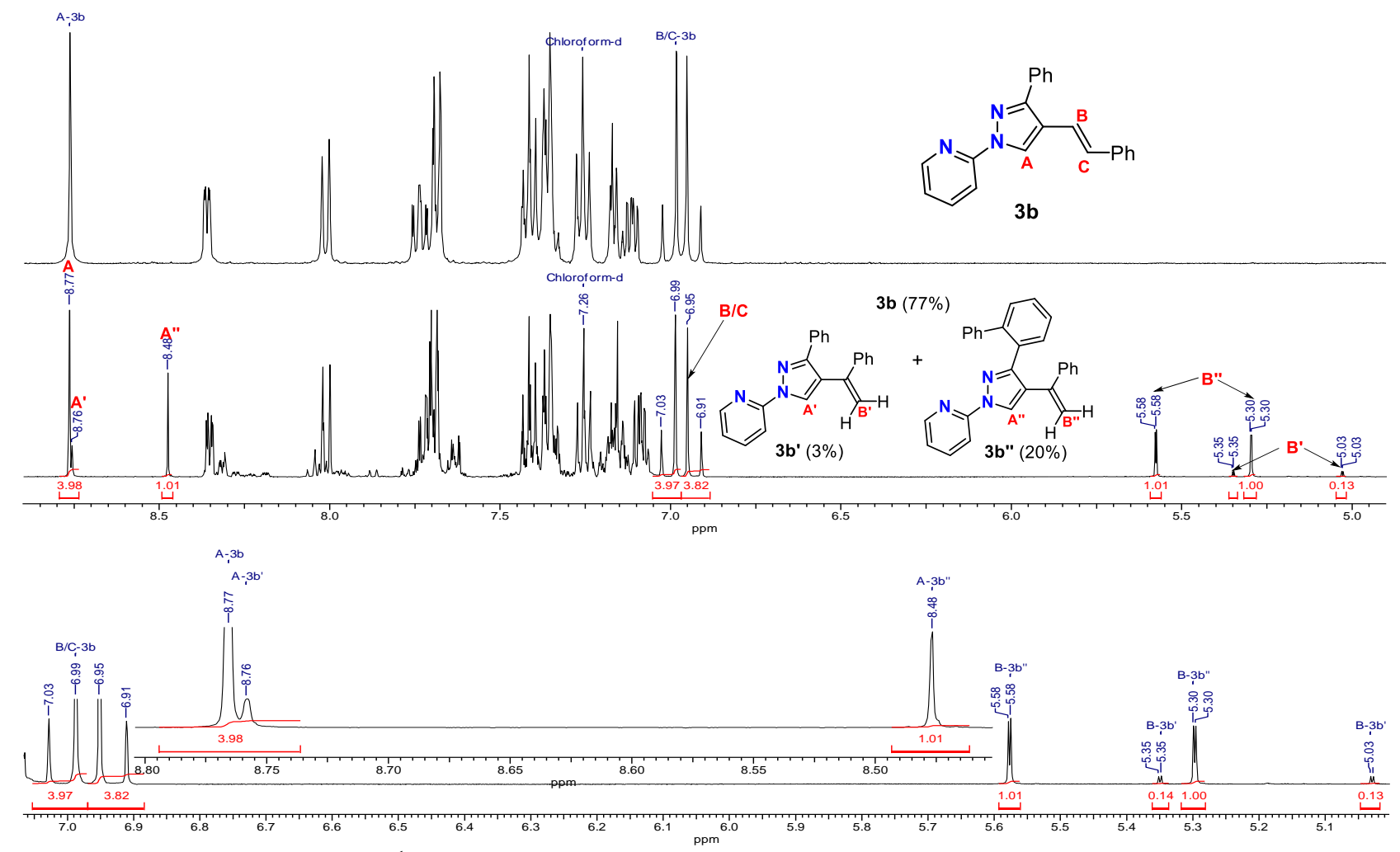

Figure S4. Expansion ${ }^{1} \mathrm{H}$ NMR spectra for the 4-styrylpyrazoles $\mathbf{3 b}$ and byproducts $\mathbf{3} \mathbf{b}$ ' and $\mathbf{3} \mathbf{b}^{\text {". }}$

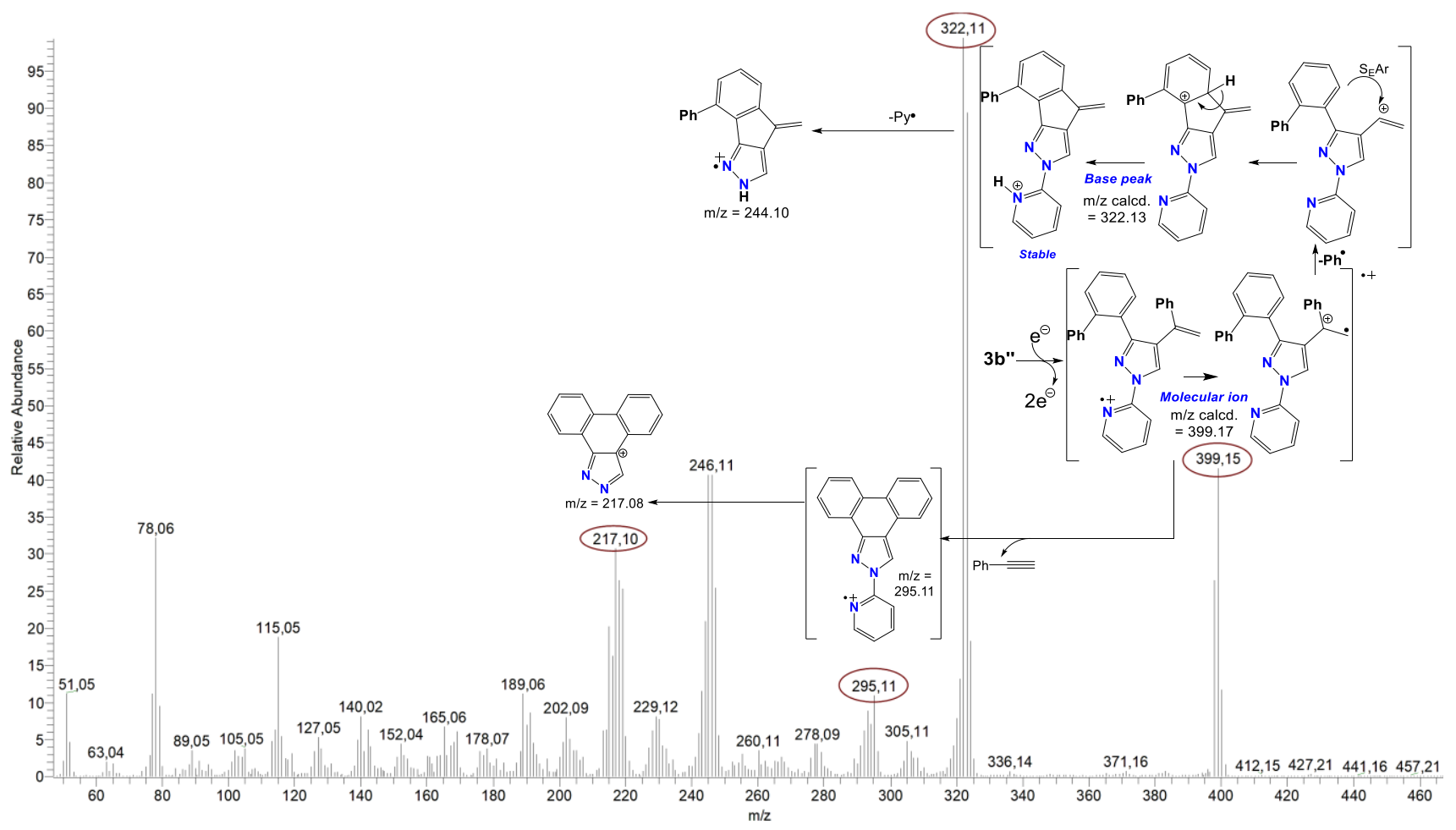

Figure S5. MS (EI) spectrum of the byproduct $\mathbf{3} \mathbf{b}^{\prime \prime}$ and its possible fragmentation mechanism. 


\section{Copies of NMR spectra}

${ }^{1} \mathrm{H}$ and ${ }^{13} \mathrm{C}\left\{{ }^{1} \mathrm{H}\right\}$ NMR spectra of 2-(3-(4-nitrophenyl)-4-vinyl-1H-pyrazol-1-yl)pyridine (4a)
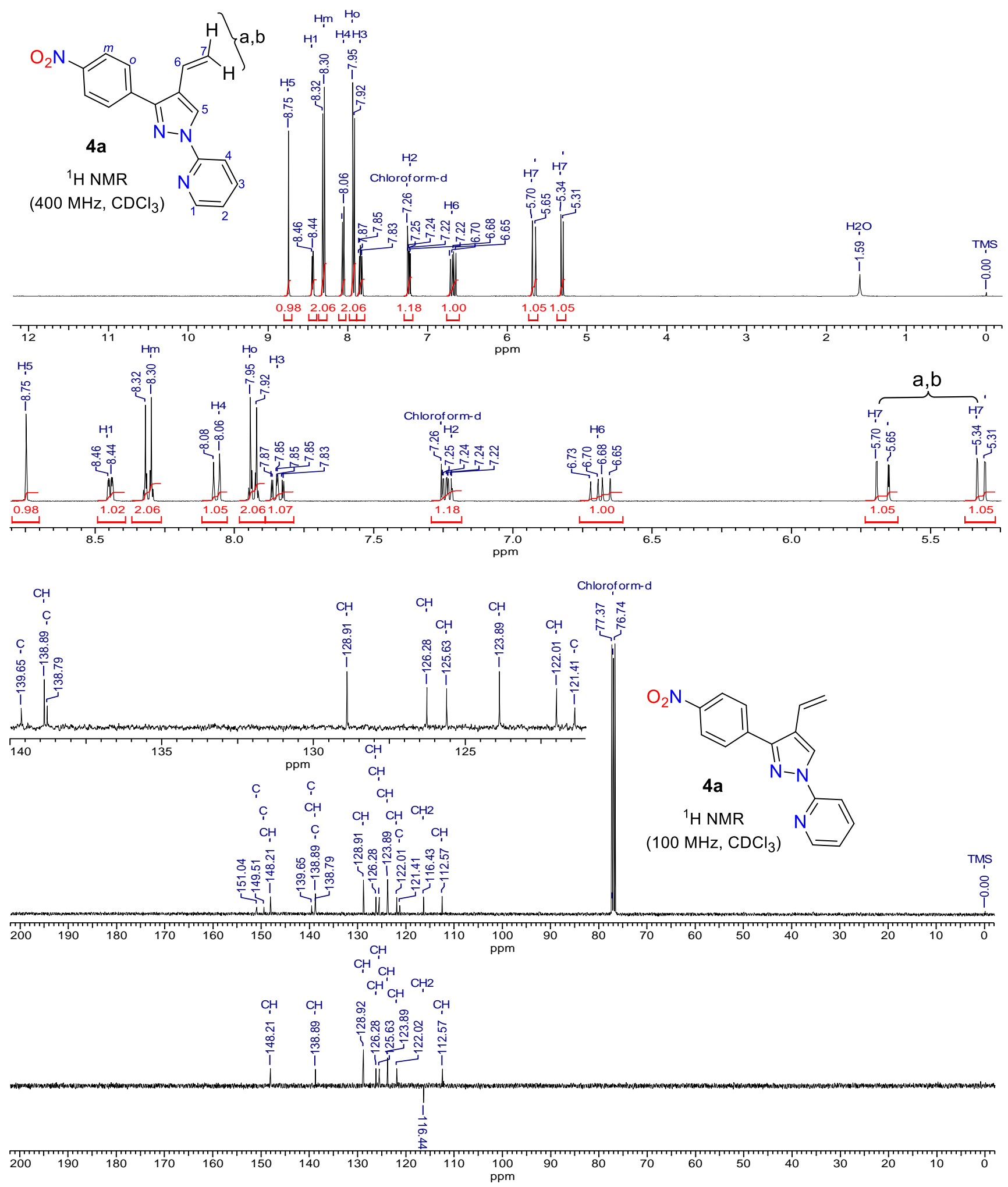
${ }^{1} \mathrm{H}$ and ${ }^{13} \mathrm{C}\left\{{ }^{1} \mathrm{H}\right\}$ NMR spectra of 2-(3-phenyl-4-vinyl-1H-pyrazol-1-yl)pyridine (4b)
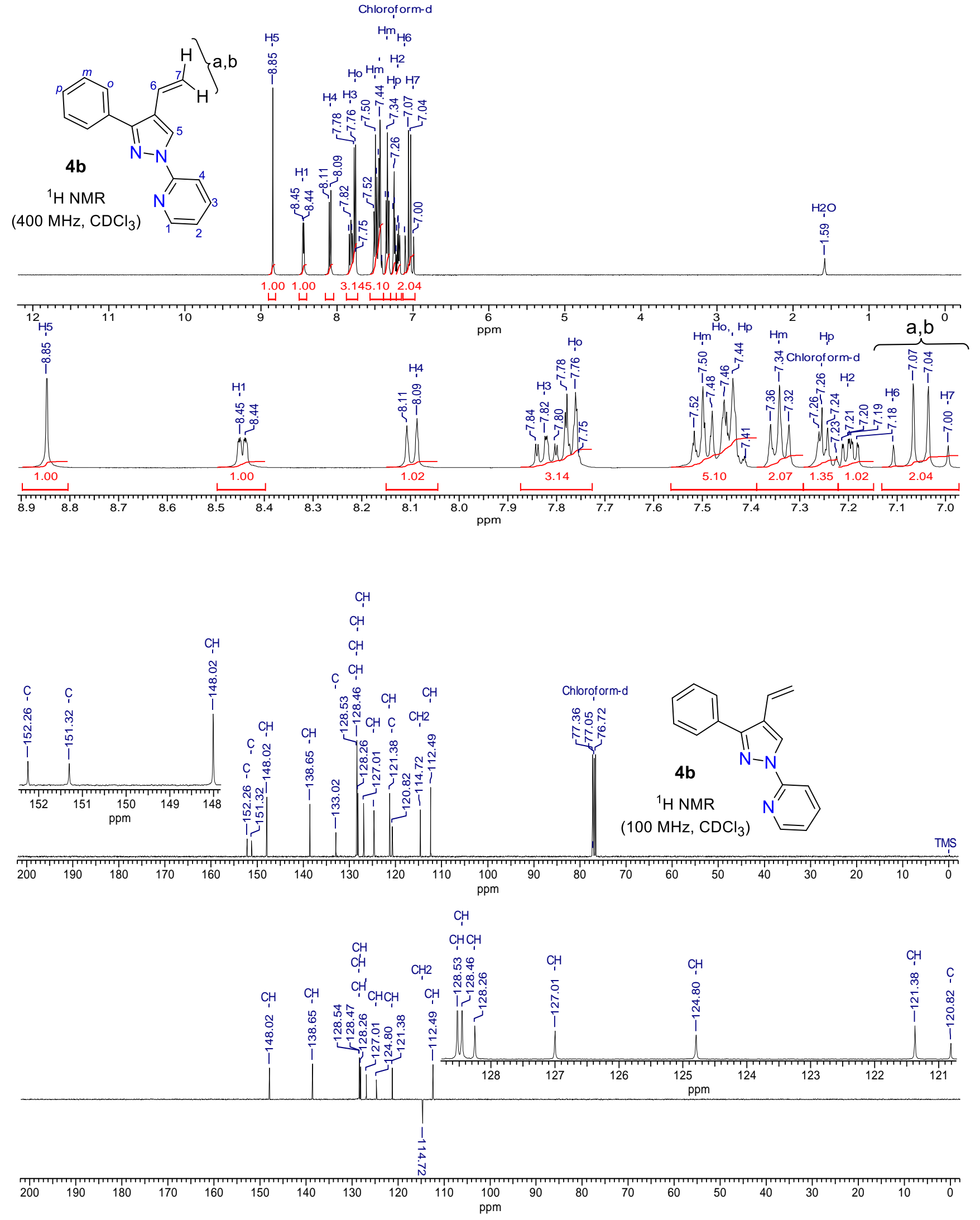
${ }^{1} \mathrm{H}$ and ${ }^{13} \mathrm{C}\left\{{ }^{1} \mathrm{H}\right\}$ NMR spectra of 2-(3-(4-methoxyphenyl)-4-vinyl-1H-pyrazol-1-yl)pyridine (4c)
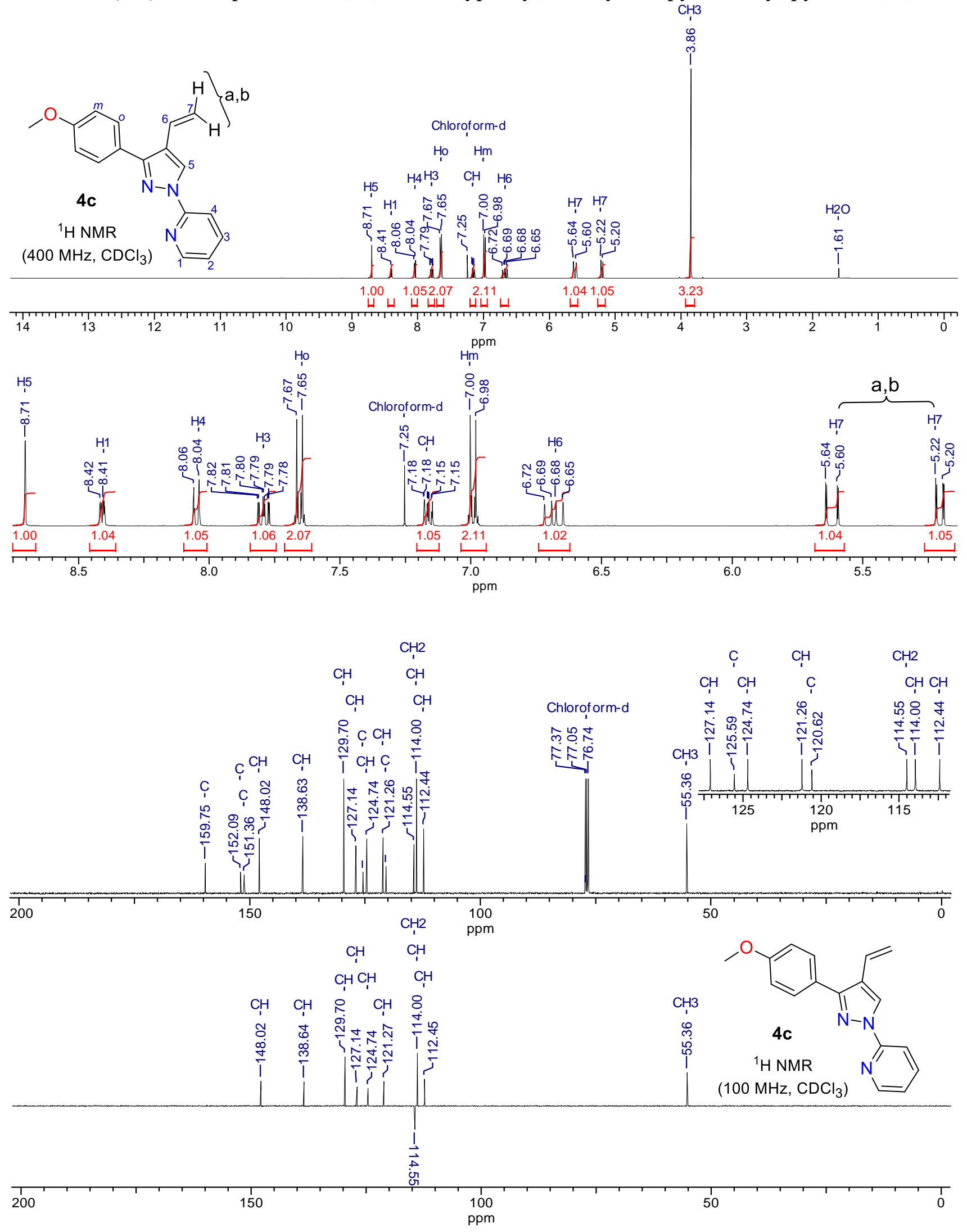
${ }^{1} \mathrm{H}$ and ${ }^{13} \mathrm{C}\left\{{ }^{1} \mathrm{H}\right\}$ NMR spectra of 1,3-diphenyl-4-vinyl-1 $H$-pyrazole (4d)
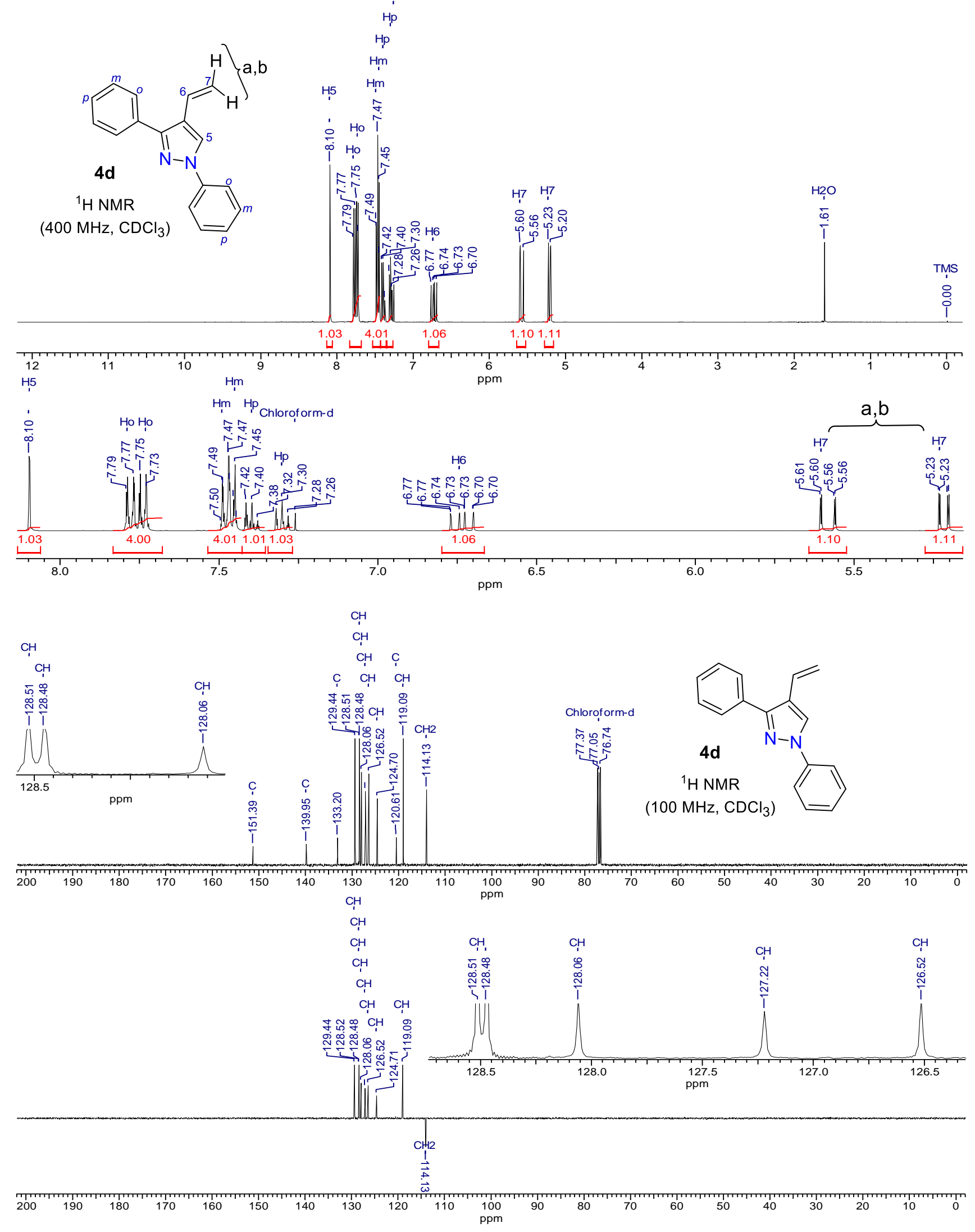

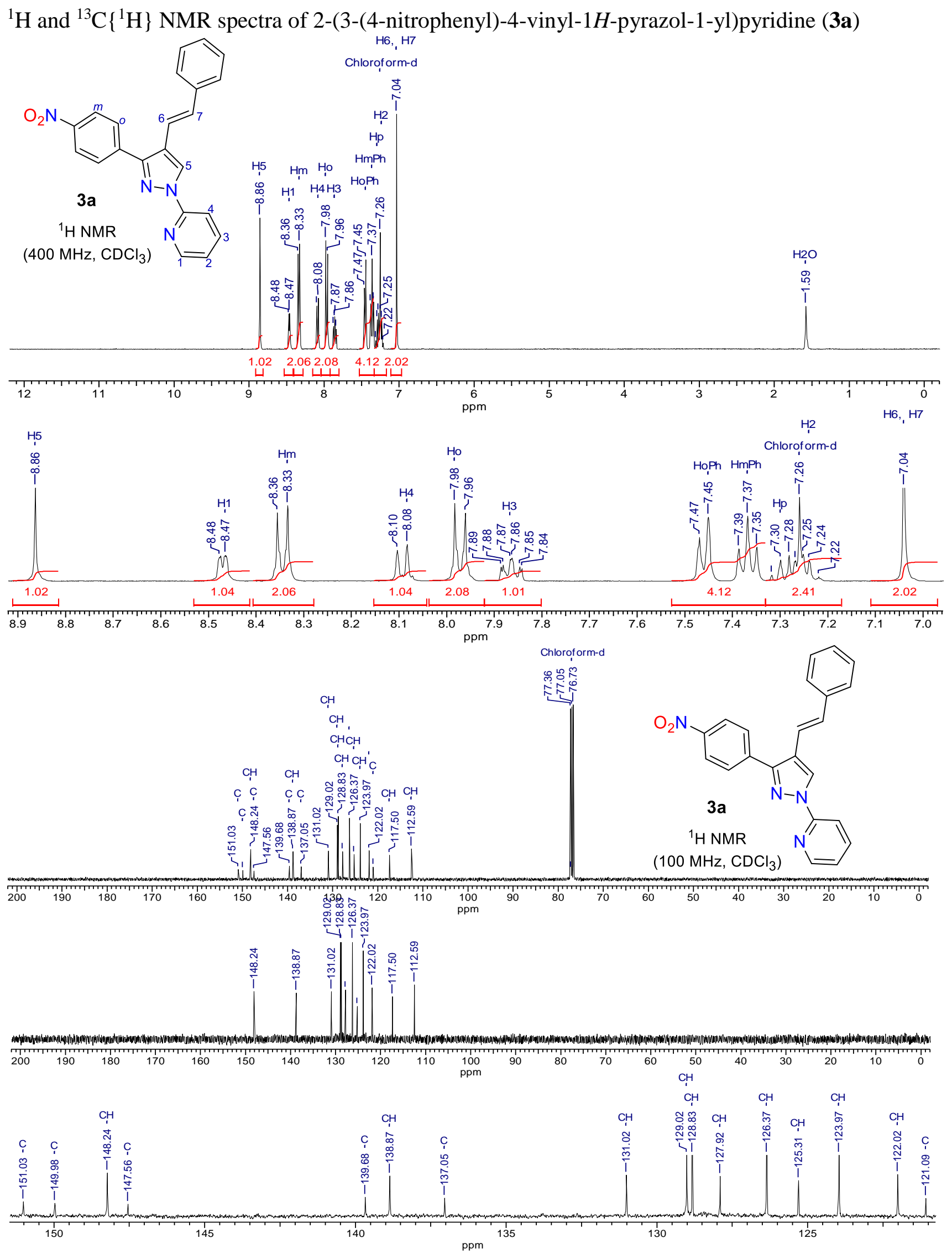
${ }^{1} \mathrm{H}$ and ${ }^{13} \mathrm{C}\left\{{ }^{1} \mathrm{H}\right\}$ NMR spectra of $(E)$-2-(3-phenyl-4-styryl-1H-pyrazol-1-yl)pyridine (3b)
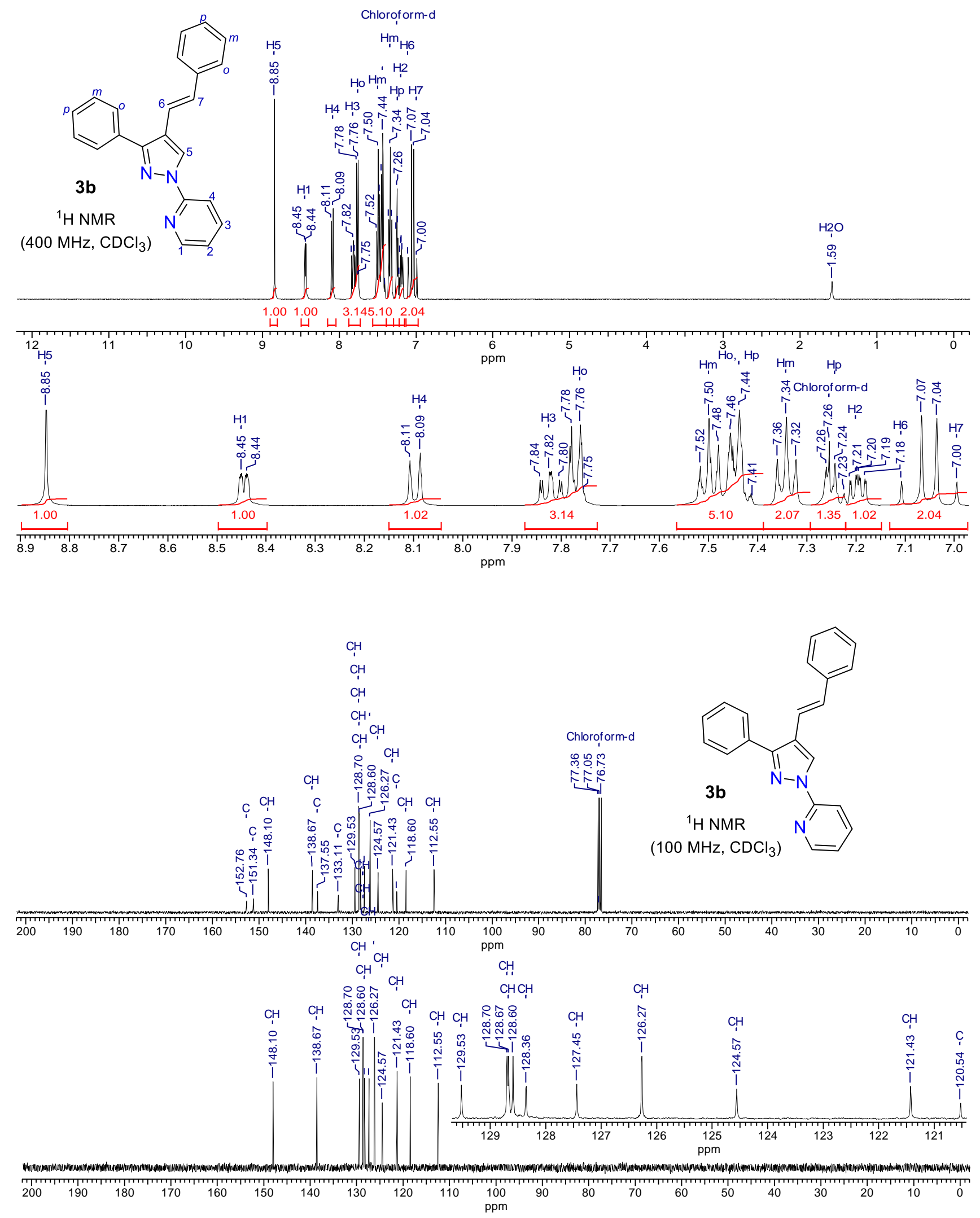
HSQC and HMBC spectra of (E)-2-(3-phenyl-4-styryl-1H-pyrazol-1-yl)pyridine (3b)

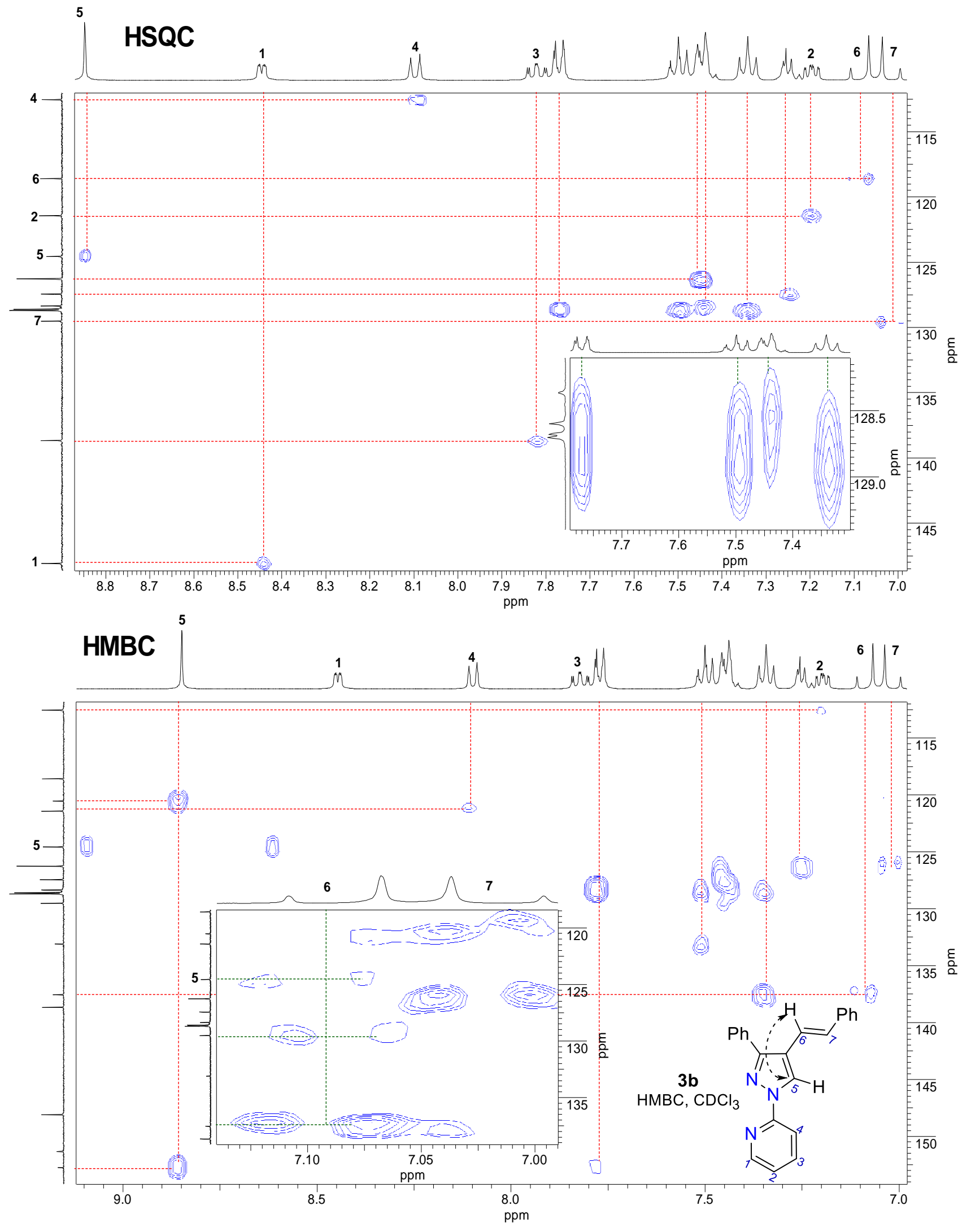


${ }^{1} \mathrm{H}$ and ${ }^{13} \mathrm{C}\left\{{ }^{1} \mathrm{H}\right\}$ NMR spectra of (E)-2-(3-(4-methoxyphenyl)-4-styryl-1H-pyrazol-1-yl)pyridine (3c)
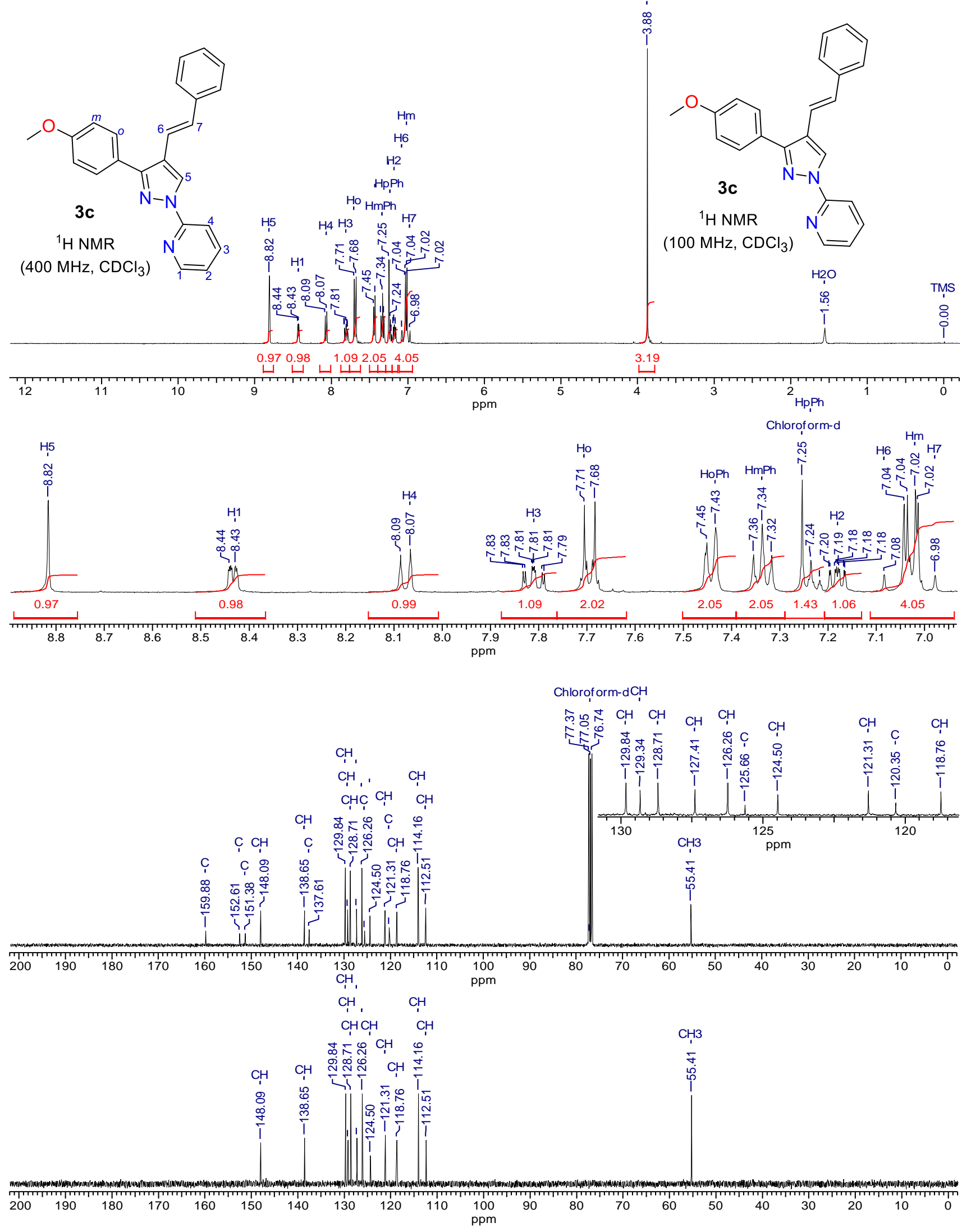
${ }^{1} \mathrm{H}$ and ${ }^{13} \mathrm{C}\left\{{ }^{1} \mathrm{H}\right\}$ NMR spectra of (E)-1,3-diphenyl-4-styryl-1H-pyrazole (3d)
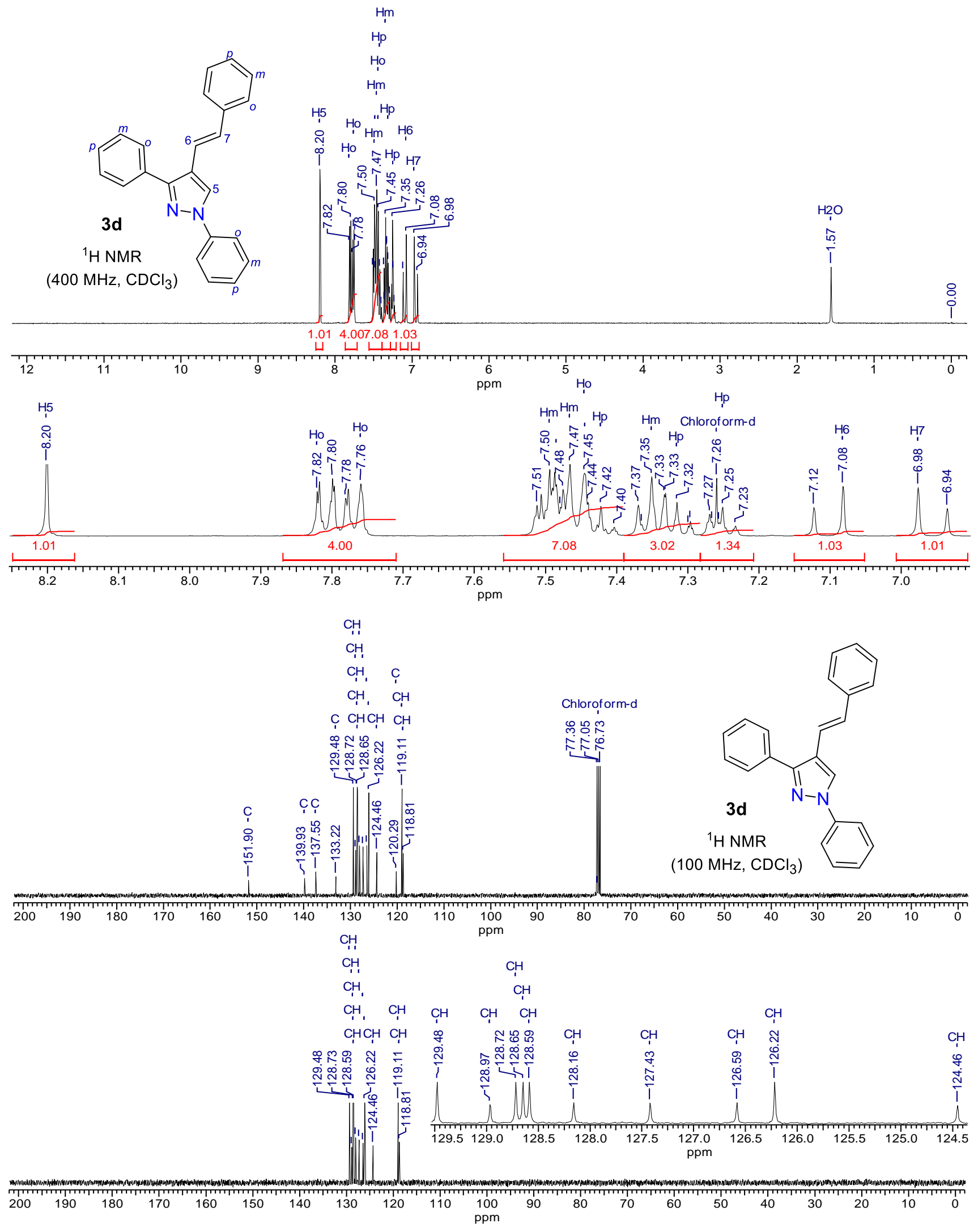


\section{HRMS analysis data of the compounds 4a-d and 3a-d}

\begin{tabular}{|l|l|l|l|l|}
\hline Compound Label & $\mathbf{m} / \boldsymbol{z}$ & RT & Algorithm & Mass \\
\hline Cpd 3: C16 H12 N4 O2 & 293.1033 & 6.391 & Find By Formula & 292.096 \\
\hline
\end{tabular}

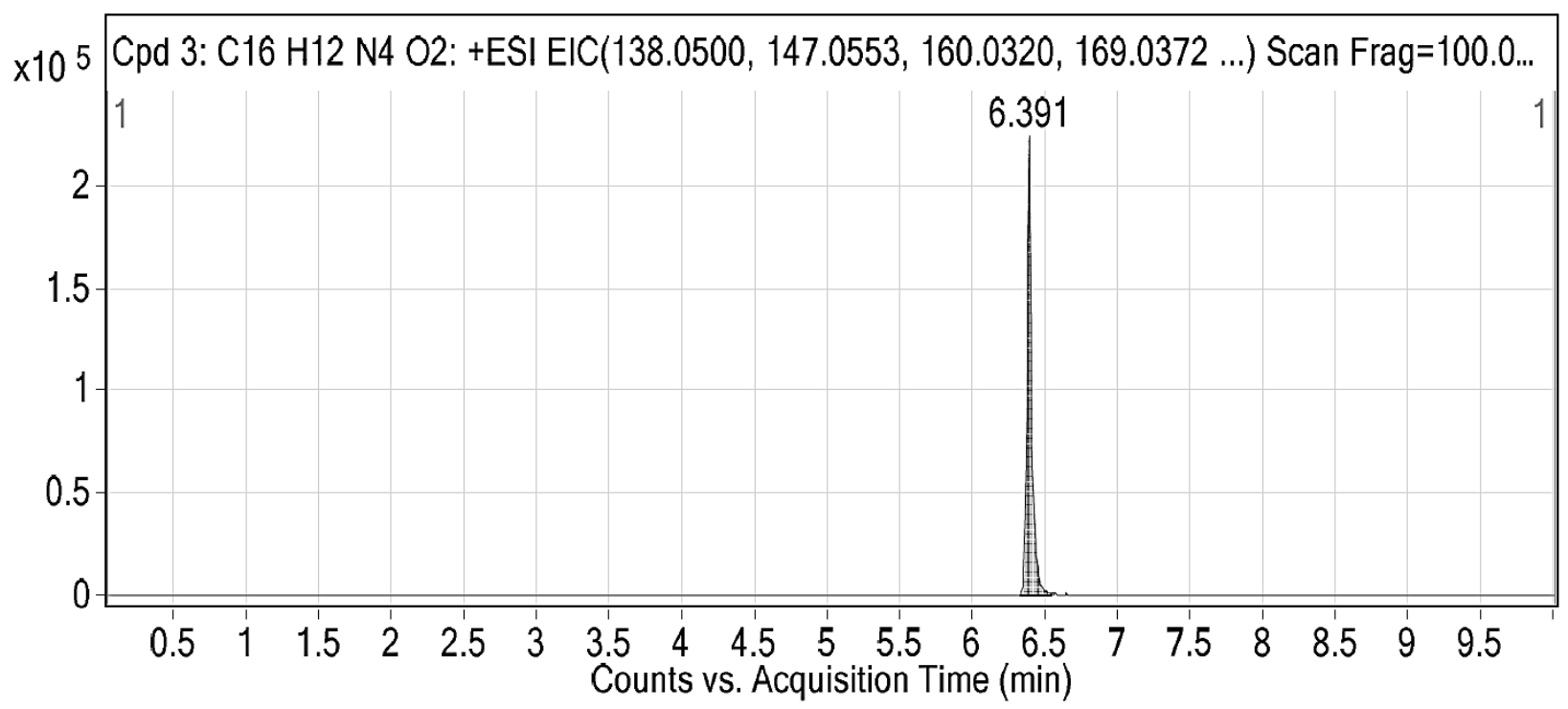

MS Spectrum

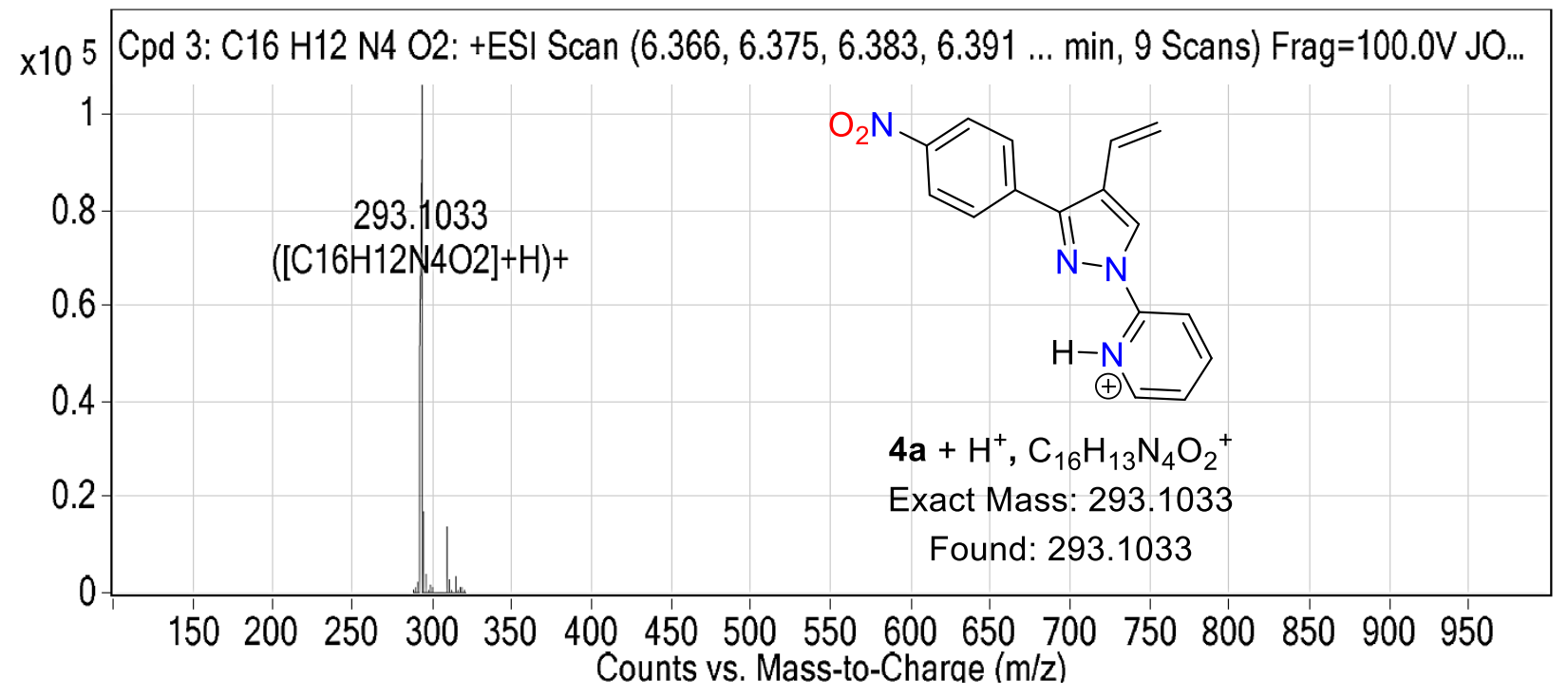

MS Spectrum Peak List

\begin{tabular}{|r|r|r|r|r|l|l|}
\hline $\boldsymbol{m} / \boldsymbol{z}$ & Calc $\boldsymbol{m} / \boldsymbol{z}$ & Diff(ppm) & $\mathbf{z}$ & Abund & Formula & Ion \\
\hline 293.1033 & 293.1033 & 0.16 & 1 & 64245.05 & $\mathrm{C} 16 \mathrm{H} 12 \mathrm{~N} 4 \mathrm{O} 2$ & $(\mathrm{M}+\mathrm{H})+$ \\
\hline 294.1059 & 294.1062 & 0.95 & 1 & 10567.85 & $\mathrm{C} 16 \mathrm{H} 12 \mathrm{~N} 4 \mathrm{O} 2$ & $(\mathrm{M}+\mathrm{H})+$ \\
\hline 295.109 & 295.1088 & -0.93 & 1 & 1225.31 & $\mathrm{C} 16 \mathrm{H} 12 \mathrm{~N} 4 \mathrm{O} 2$ & $(\mathrm{M}+\mathrm{H})+$ \\
\hline 296.1116 & 296.1112 & -1.26 & 1 & 112.54 & $\mathrm{C} 16 \mathrm{H} 12 \mathrm{~N} 4 \mathrm{O} 2$ & $(\mathrm{M}+\mathrm{H})+$ \\
\hline 315.0849 & 315.0852 & 1 & 1 & 1074.64 & $\mathrm{C16H} 12 \mathrm{~N} 4 \mathrm{O} 2$ & $(\mathrm{M}+\mathrm{Na})+$ \\
\hline 316.0888 & 316.0881 & -1.99 & 1 & 154.88 & $\mathrm{C16H} 12 \mathrm{~N} 4 \mathrm{O} 2$ & $(\mathrm{M}+\mathrm{Na})+$ \\
\hline
\end{tabular}




\begin{tabular}{|l|l|l|l|l|}
\hline Compound Label & $\boldsymbol{m} / \boldsymbol{z}$ & RT & Algorithm & Mass \\
\hline Cpd 1: C16 H13 N3 & 248.1179 & 6.312 & Find By Formula & 247.1107 \\
\hline
\end{tabular}

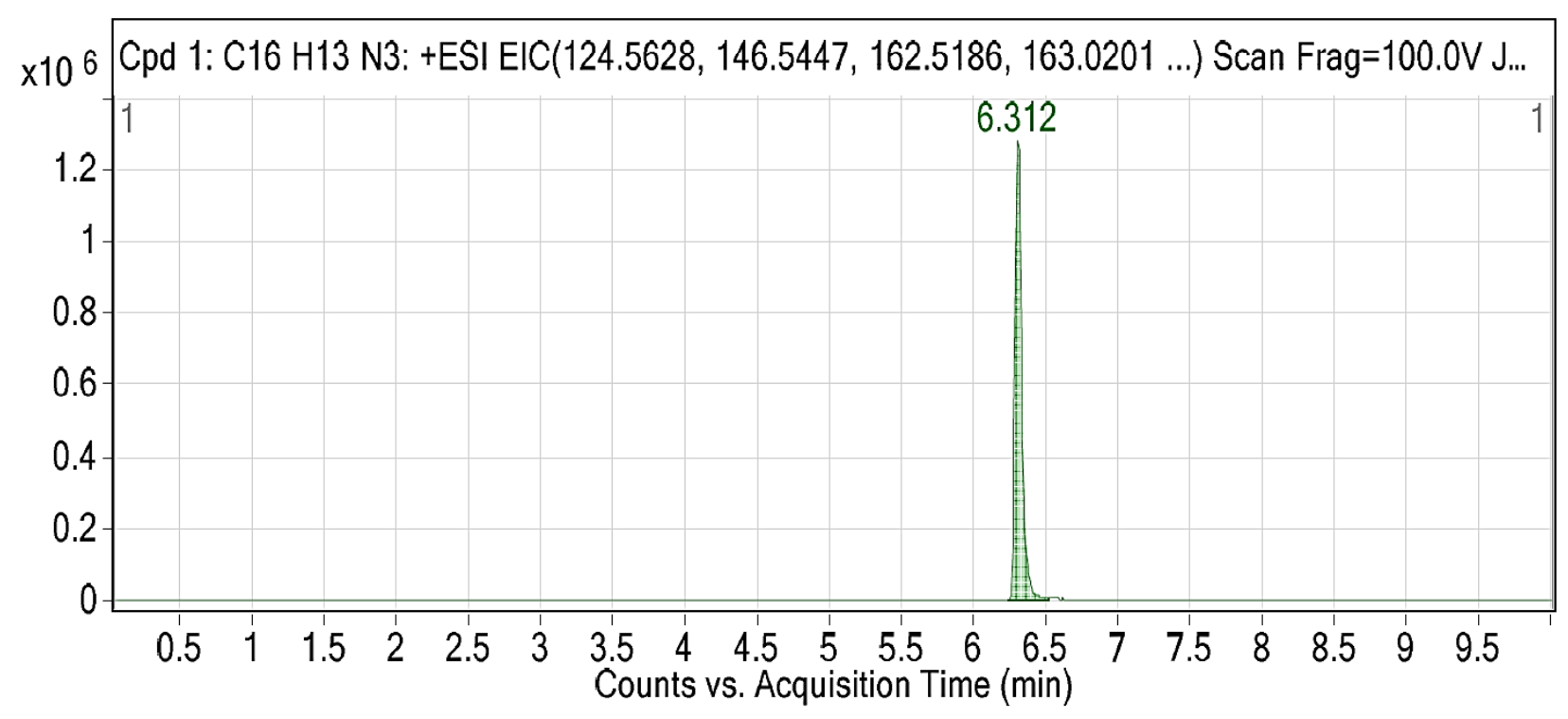

MS Spectrum

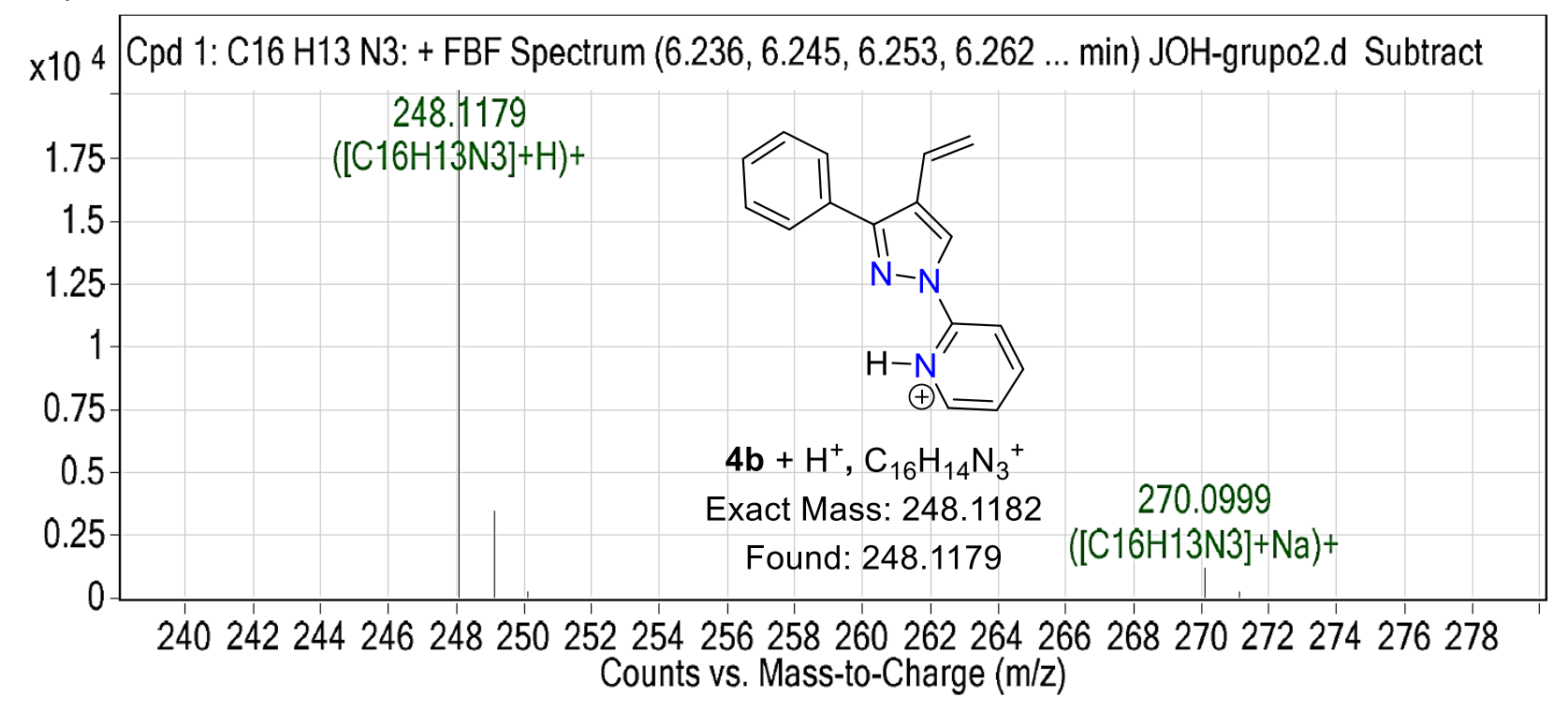

MS Spectrum Peak List

\begin{tabular}{|r|r|r|l|l|}
\hline $\boldsymbol{m} / \boldsymbol{z}$ & $\mathbf{z}$ & Abund & Formula & Ion \\
\hline 248.1179 & 1 & 20149.52 & C16H13N3 & $(\mathrm{M}+\mathrm{H})+$ \\
\hline 249.1212 & 1 & 3391.14 & $\mathrm{C} 16 \mathrm{H} 13 \mathrm{~N} 3$ & $(\mathrm{M}+\mathrm{H})+$ \\
\hline 250.1241 & 1 & 248.98 & $\mathrm{C} 16 \mathrm{H} 13 \mathrm{~N} 3$ & $(\mathrm{M}+\mathrm{H})+$ \\
\hline 270.0999 & 1 & 1117.43 & $\mathrm{C} 16 \mathrm{H} 13 \mathrm{~N} 3$ & $(\mathrm{M}+\mathrm{Na})+$ \\
\hline 271.1029 & 1 & 190.17 & C16H13N3 & $(\mathrm{M}+\mathrm{Na})+$ \\
\hline
\end{tabular}




\begin{tabular}{|l|l|l|l|l|}
\hline Compound Label & $\mathbf{m} / \boldsymbol{z}$ & RT & Algorithm & Mass \\
\hline Cpd 3: C17 H15 N3 O & 278.1285 & 6.236 & Find By Formula & 277.1213 \\
\hline
\end{tabular}

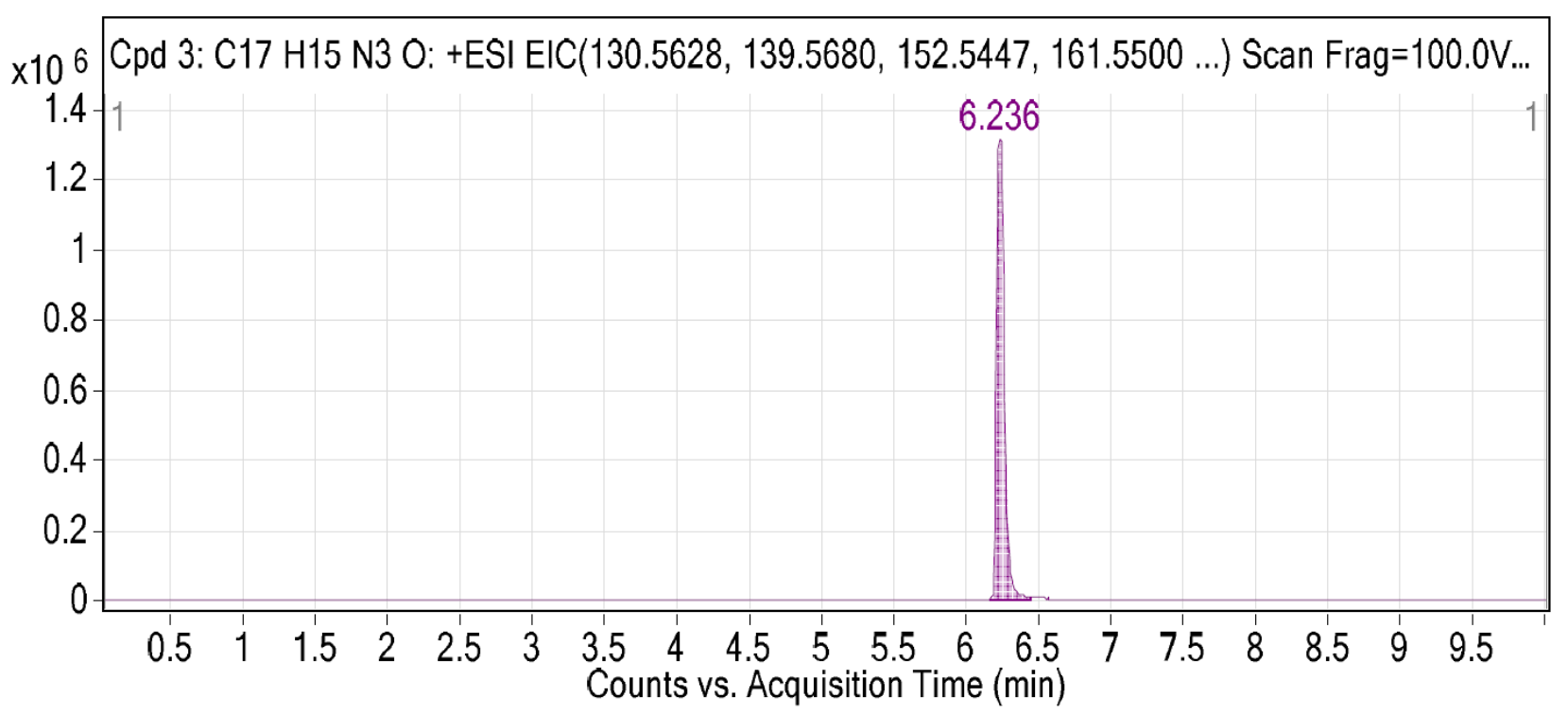

MS Spectrum

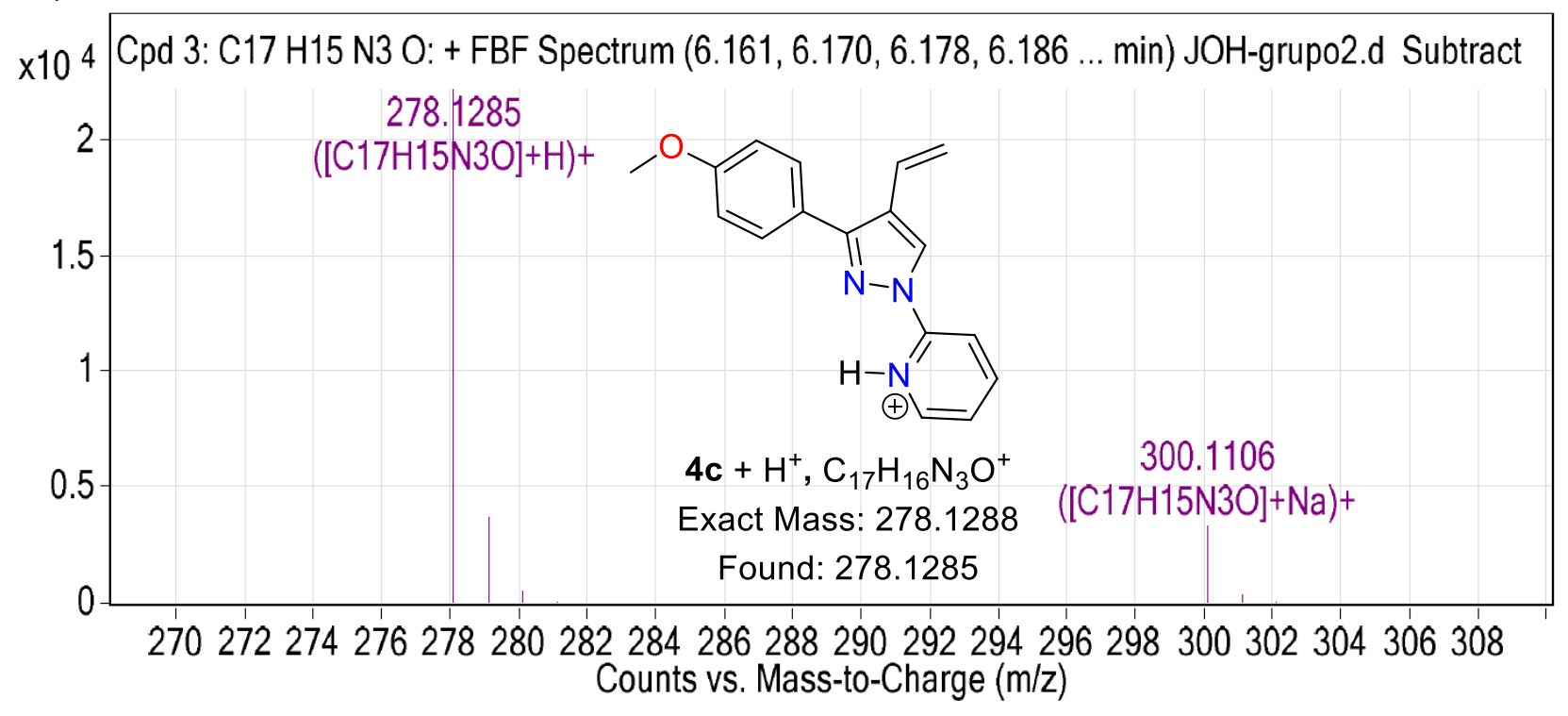

MS Spectrum Peak List

\begin{tabular}{|r|r|r|l|l|}
\hline $\boldsymbol{m} / \boldsymbol{z}$ & $\mathbf{z}$ & \multicolumn{1}{l|}{ abund } & Formula & Ion \\
\hline 278.1285 & 1 & 22170.8 & C17H15N3O & $(\mathrm{M}+\mathrm{H})+$ \\
\hline 279.1318 & 1 & 3613.07 & C17H15N3O & $(\mathrm{M}+\mathrm{H})+$ \\
\hline 280.1351 & 1 & 432.39 & C17H15N3O & $(\mathrm{M}+\mathrm{H})+$ \\
\hline 281.1364 & 1 & 24.62 & C17H15N3O & $(\mathrm{M}+\mathrm{H})+$ \\
\hline 300.1106 & 1 & 3281.81 & C17H15N3O & $(\mathrm{M}+\mathrm{Na})+$ \\
\hline 301.1126 & 1 & 329.61 & C17H15N3O & $(\mathrm{M}+\mathrm{Na})+$ \\
\hline 302.1182 & 1 & 26.7 & C17H15N3O & $(\mathrm{M}+\mathrm{Na})+$ \\
\hline
\end{tabular}




\begin{tabular}{|l|l|l|l|l|}
\hline Compound Label & $\boldsymbol{m} / \boldsymbol{z}$ & RT & Algorithm & Mass \\
\hline Cpd 1: C17 H14 N2 & 247.1227 & 6.408 & Find By Formula & 246.1154 \\
\hline
\end{tabular}

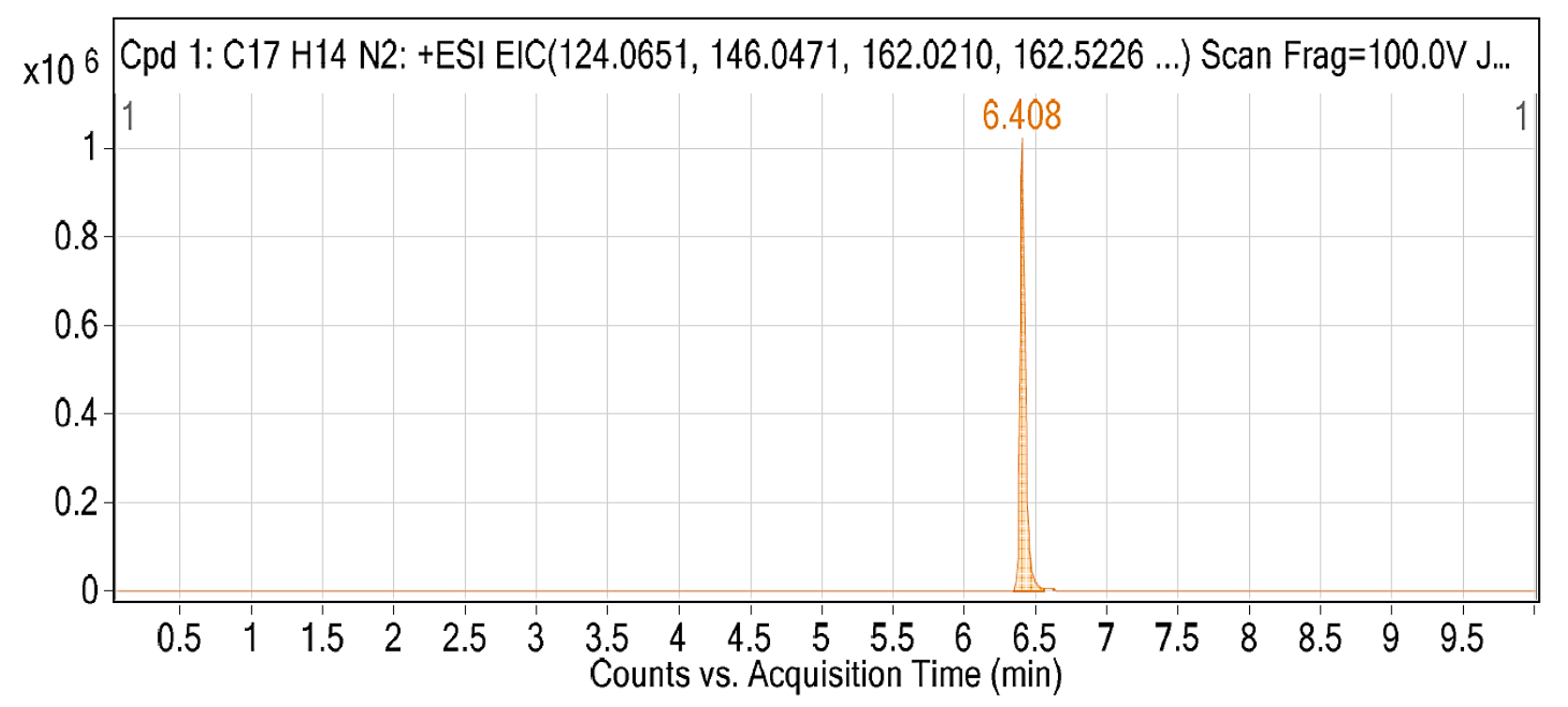

MS Spectrum

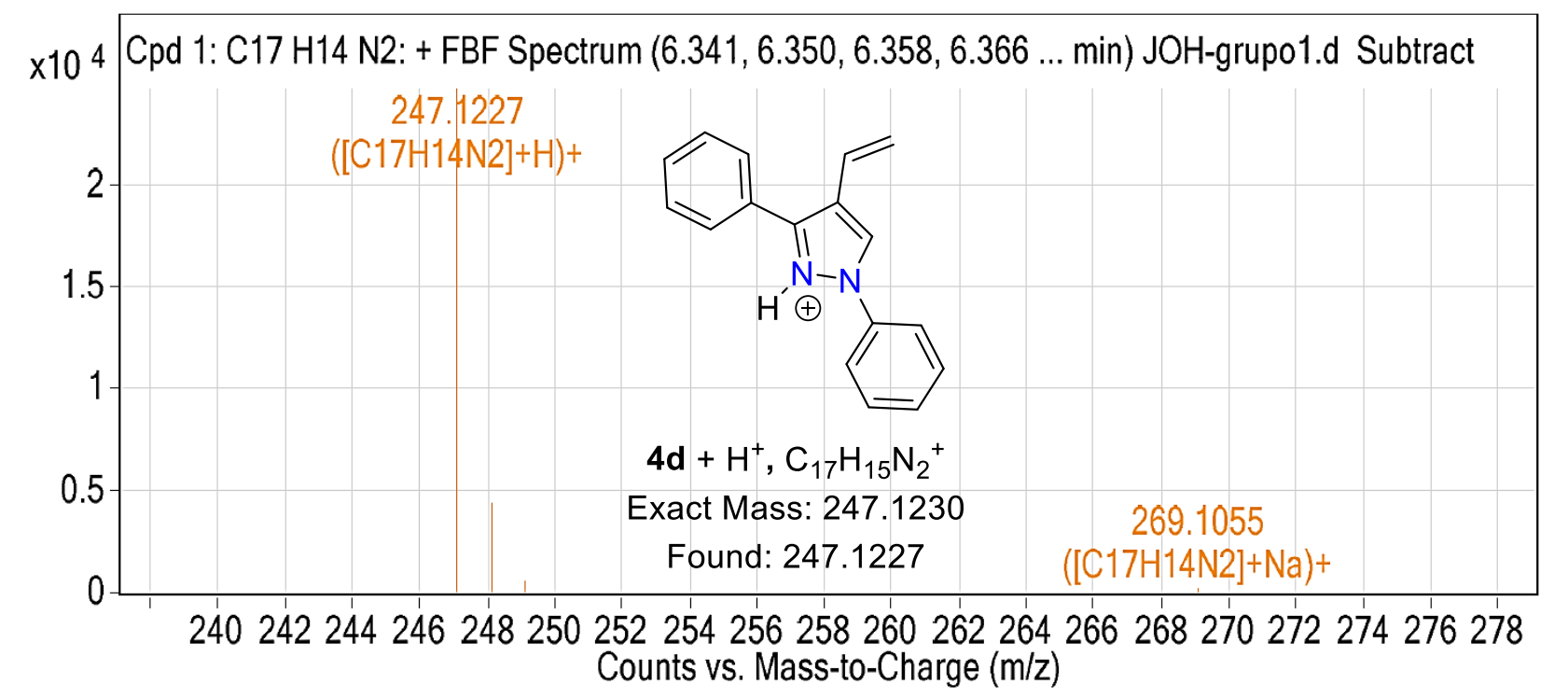

MS Spectrum Peak List

\begin{tabular}{|r|r|l|l|l|}
\hline $\boldsymbol{m} / \boldsymbol{z}$ & $\mathbf{z}$ & \multicolumn{1}{|l|}{ Abund } & Formula & Ion \\
\hline 247.1227 & 1 & 24645.32 & C17H14N2 & $(\mathrm{M}+\mathrm{H})+$ \\
\hline 248.1258 & 1 & 4332.52 & C17H14N2 & $(\mathrm{M}+\mathrm{H})+$ \\
\hline 249.1314 & 1 & 485.64 & C17H14N2 & $(\mathrm{M}+\mathrm{H})+$ \\
\hline 269.1055 & 1 & 106.4 & C17H14N2 & $(\mathrm{M}+\mathrm{Na})+$ \\
\hline
\end{tabular}




\begin{tabular}{|l|l|l|l|l|}
\hline Compound Label & $\boldsymbol{m} / \boldsymbol{z}$ & RT & Algorithm & Mass \\
\hline Cpd 4: C22 H16 N4 O2 & 369.134 & 7.118 & Find By Formula & 368.1268 \\
\hline
\end{tabular}

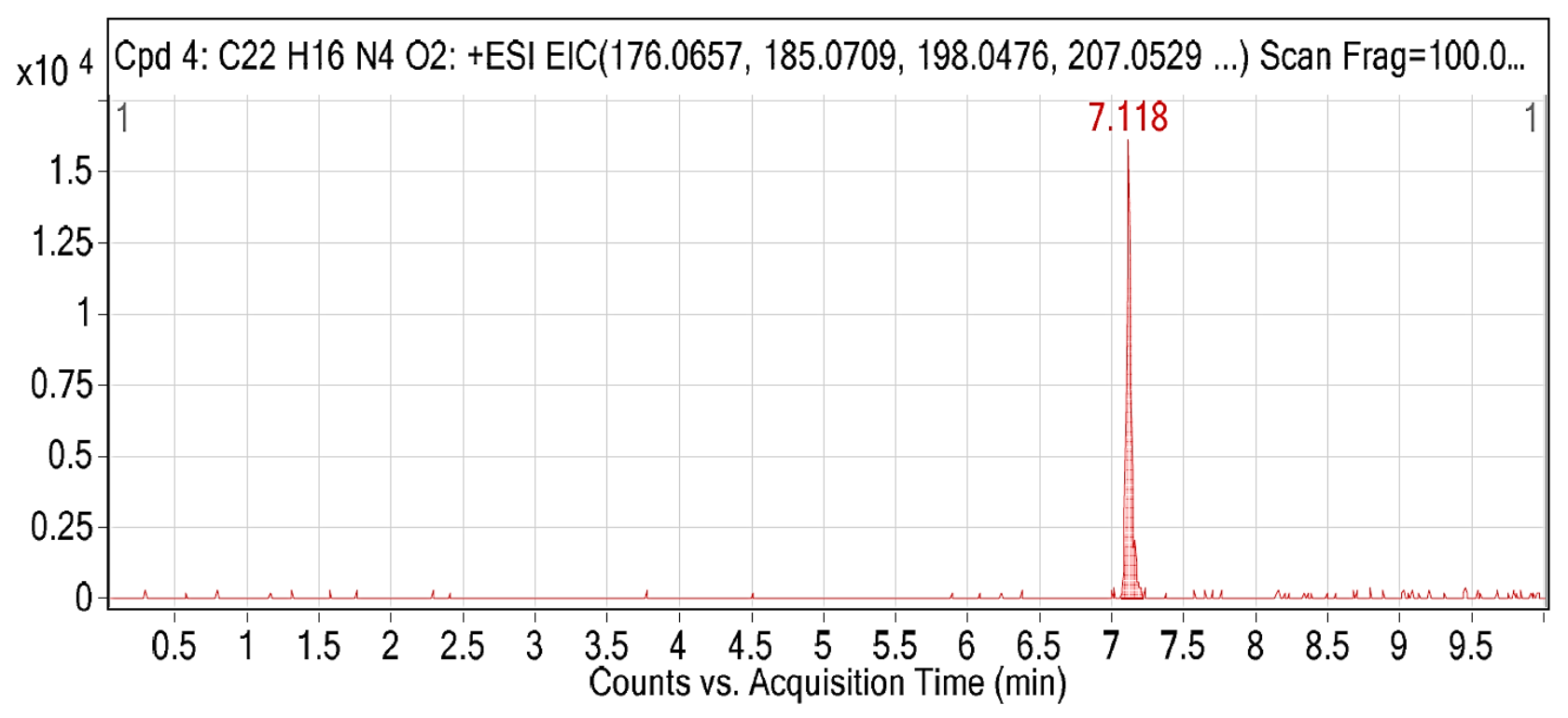

MS Spectrum

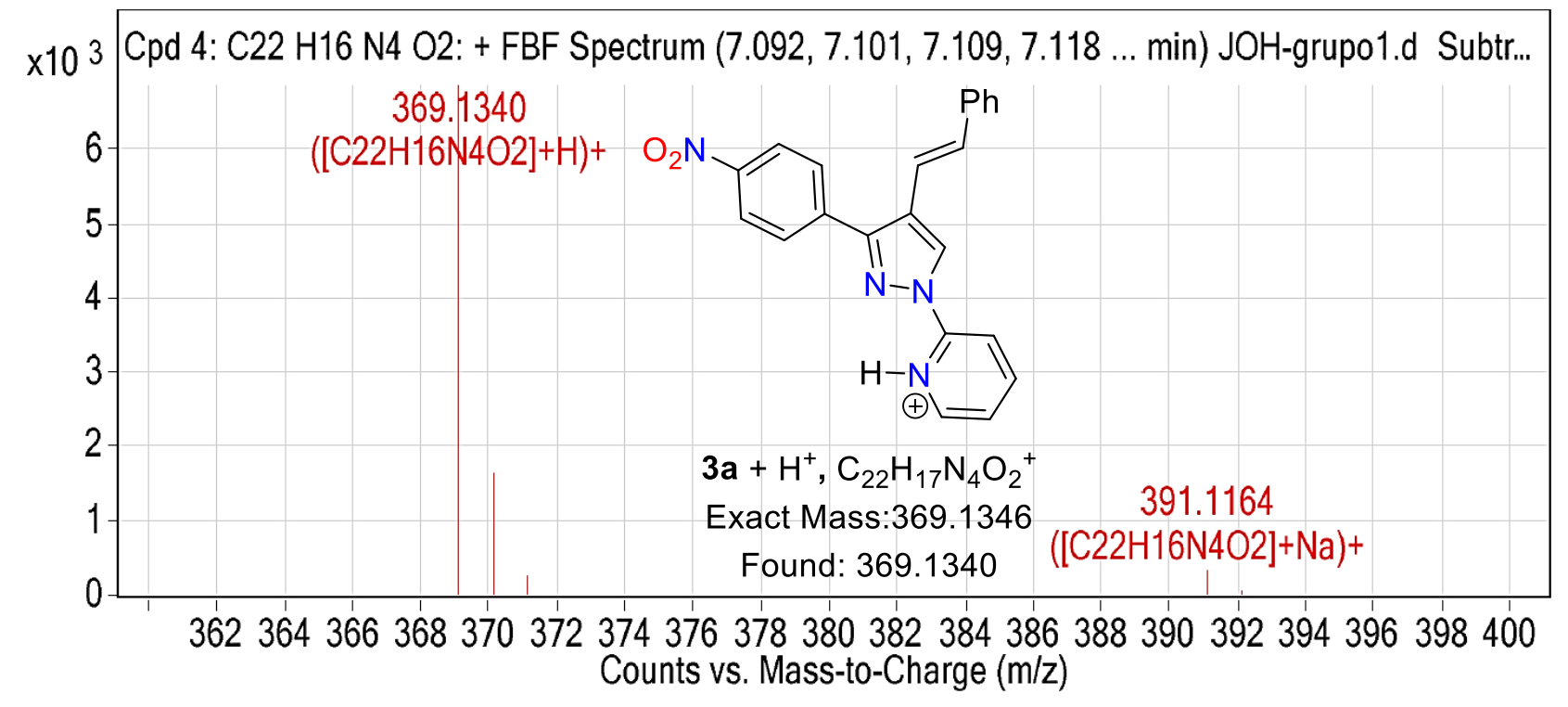

MS Spectrum Peak List

\begin{tabular}{|r|r|r|l|l|}
\hline $\boldsymbol{m} / \boldsymbol{z}$ & $\boldsymbol{z}$ & Abund & Formula & Ion \\
\hline 369.134 & 1 & 6864.31 & $\mathrm{C} 22 \mathrm{H} 16 \mathrm{~N} 4 \mathrm{O} 2$ & $(\mathrm{M}+\mathrm{H})+$ \\
\hline 370.1369 & 1 & 1637.32 & $\mathrm{C} 22 \mathrm{H} 16 \mathrm{~N} 4 \mathrm{O} 2$ & $(\mathrm{M}+\mathrm{H})+$ \\
\hline 371.1423 & 1 & 232.81 & $\mathrm{C} 22 \mathrm{H} 16 \mathrm{~N} 4 \mathrm{O} 2$ & $(\mathrm{M}+\mathrm{H})+$ \\
\hline 391.1164 & 1 & 313.1 & $\mathrm{C} 22 \mathrm{H} 16 \mathrm{~N} 4 \mathrm{O} 2$ & $(\mathrm{M}+\mathrm{Na})+$ \\
\hline 392.1186 & 1 & 49.29 & $\mathrm{C} 22 \mathrm{H} 16 \mathrm{~N} 4 \mathrm{O} 2$ & $(\mathrm{M}+\mathrm{Na})+$ \\
\hline
\end{tabular}




\begin{tabular}{|l|l|l|l|l|}
\hline Compound Label & $\mathbf{m} / \boldsymbol{z}$ & RT & Algorithm & Mass \\
\hline Cpd 2: C22 H17 N3 & 324.1488 & 7.096 & Find By Formula & 323.1415 \\
\hline
\end{tabular}

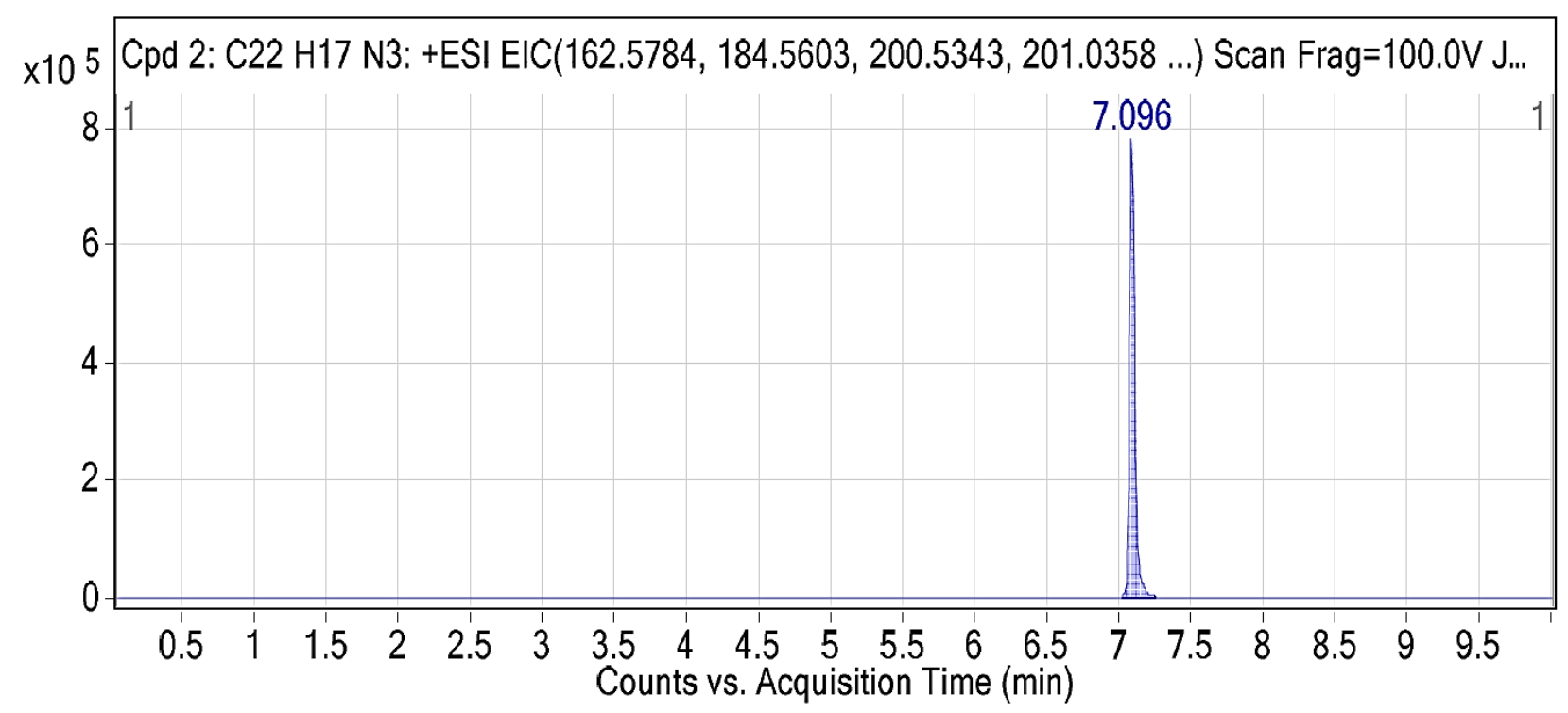

MS Spectrum

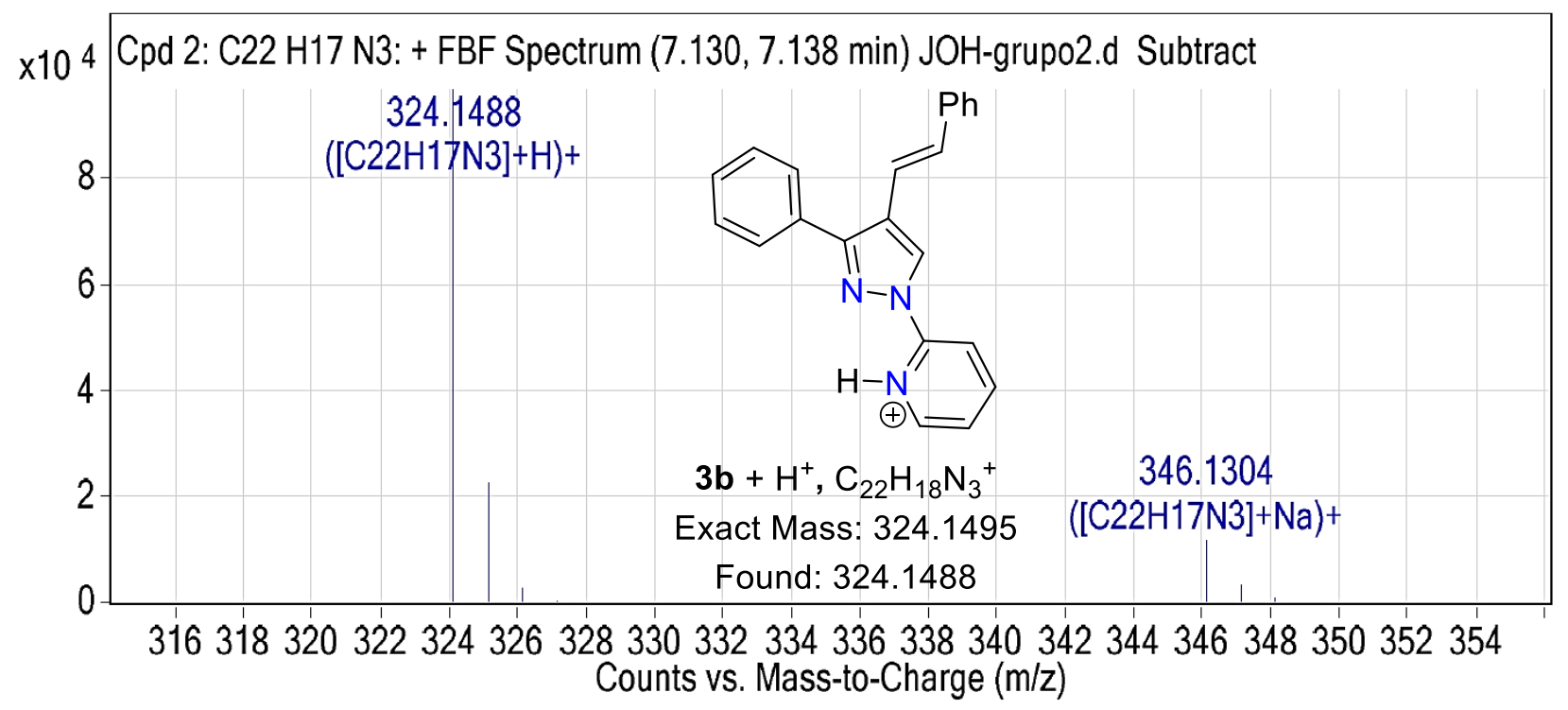

MS Spectrum Peak List

\begin{tabular}{|r|r|r|l|l|}
\hline $\boldsymbol{m} / \boldsymbol{z}$ & $\mathbf{z}$ & \multicolumn{1}{|l|}{ abund } & Formula & Ion \\
\hline 324.1488 & 1 & 96625.05 & C22H17N3 & $(\mathrm{M}+\mathrm{H})+$ \\
\hline 325.1519 & 1 & 22365.43 & C22H17N3 & $(\mathrm{M}+\mathrm{H})+$ \\
\hline 326.1554 & 1 & 2545.01 & C22H17N3 & $(\mathrm{M}+\mathrm{H})+$ \\
\hline 327.1547 & 1 & 131.34 & C22H17N3 & $(\mathrm{M}+\mathrm{H})+$ \\
\hline 346.1304 & 1 & 11337.07 & C22H17N3 & $(\mathrm{M}+\mathrm{Na})+$ \\
\hline 347.1342 & 1 & 2819.75 & C22H17N3 & $(\mathrm{M}+\mathrm{Na})+$ \\
\hline 348.1359 & 1 & 393.12 & C22H17N3 & $(\mathrm{M}+\mathrm{Na})+$ \\
\hline
\end{tabular}




\begin{tabular}{|l|l|l|l|l|}
\hline Compound Label & $\mathbf{m} / \boldsymbol{z}$ & RT & Algorithm & Mass \\
\hline Cpd 4: C23 H19 N3 O & 354.1595 & 7.013 & Find By Formula & 353.1522 \\
\hline
\end{tabular}
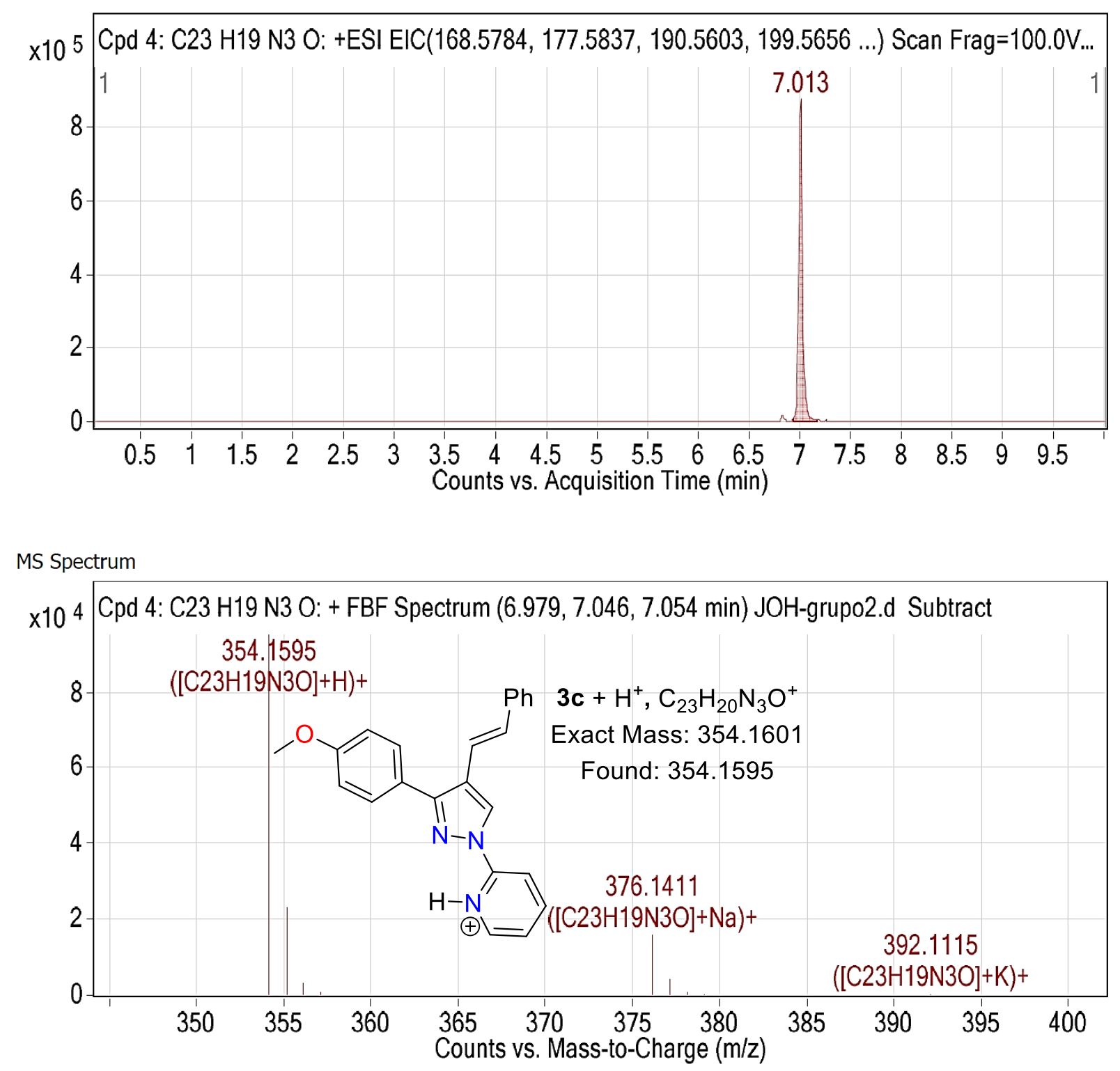

MS Spectrum Peak List

\begin{tabular}{|r|r|r|l|l|}
\hline $\boldsymbol{m} / \boldsymbol{z}$ & $\boldsymbol{z}$ & Abund & Formula & Ion \\
\hline 354.1595 & 1 & 94977.18 & C23H19N3O & $(\mathrm{M}+\mathrm{H})+$ \\
\hline 355.1627 & 1 & 23091.83 & C23H19N3O & $(\mathrm{M}+\mathrm{H})+$ \\
\hline 356.1662 & 1 & 2944.13 & C23H19N3O & $(\mathrm{M}+\mathrm{H})+$ \\
\hline 357.1631 & 1 & 470.41 & $\mathrm{C} 23 \mathrm{H} 19 \mathrm{~N} 30$ & $(\mathrm{M}+\mathrm{H})+$ \\
\hline 376.1411 & 1 & 15628.29 & C23H19N3O & $(\mathrm{M}+\mathrm{Na})+$ \\
\hline 377.1445 & 1 & 3842.84 & C23H19N3O & $(\mathrm{M}+\mathrm{Na})+$ \\
\hline 378.1465 & 1 & 480.26 & C23H19N3O & $(\mathrm{M}+\mathrm{Na})+$ \\
\hline 379.1406 & 1 & 69.06 & $\mathrm{C} 23 \mathrm{H} 19 \mathrm{~N} 30$ & $(\mathrm{M}+\mathrm{Na})+$ \\
\hline 392.1115 & 1 & 91.37 & $\mathrm{C} 23 \mathrm{H} 19 \mathrm{~N} 30$ & $(\mathrm{M}+\mathrm{K})+$ \\
\hline
\end{tabular}




\begin{tabular}{|l|l|l|l|l|}
\hline Compound Label & $\mathbf{m} / \boldsymbol{z}$ & RT & Algorithm & Mass \\
\hline Cpd 2: C23 H18 N2 & 323.1535 & 7.092 & Find By Formula & 322.1463 \\
\hline
\end{tabular}

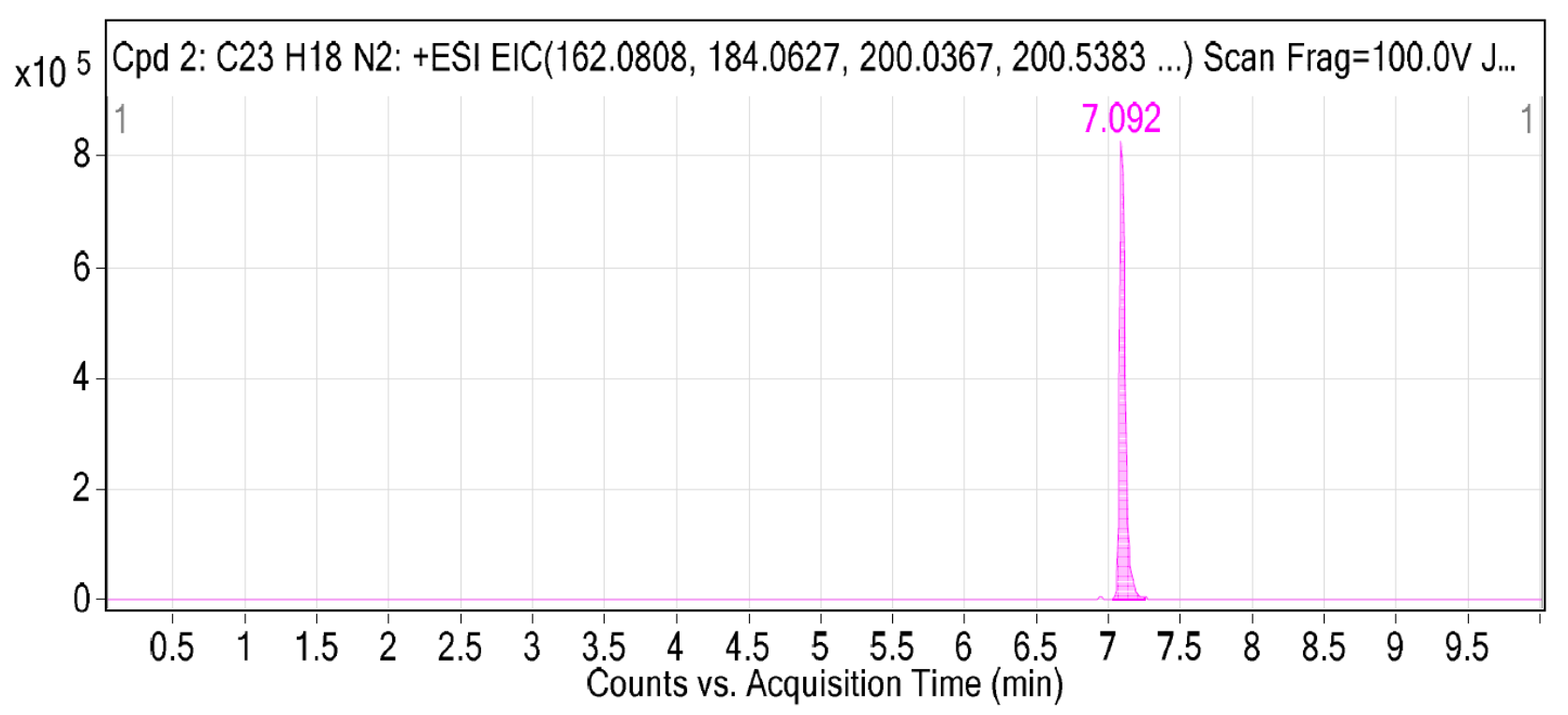

MS Spectrum

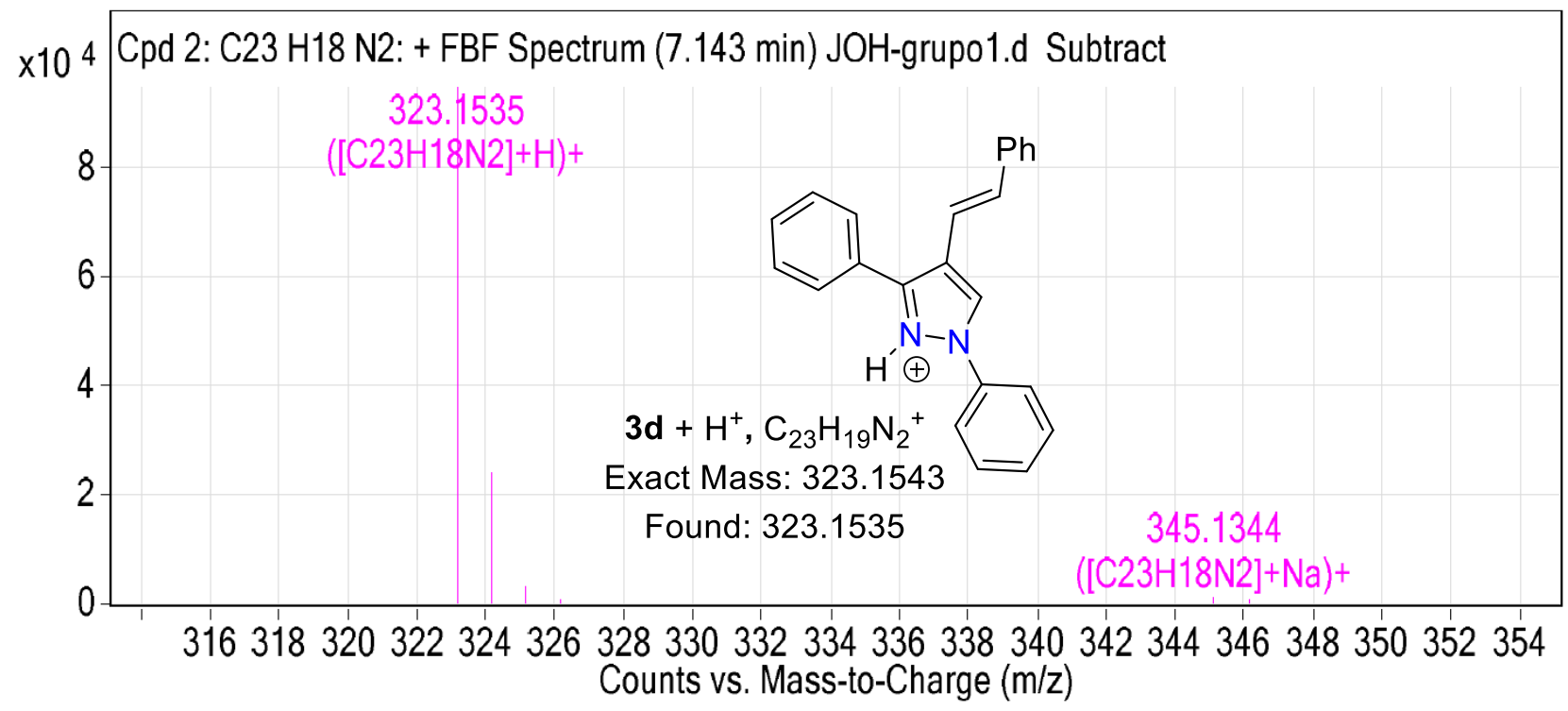

MS Spectrum Peak List

\begin{tabular}{|r|r|r|l|l|}
\hline $\boldsymbol{m} / \boldsymbol{z}$ & $\mathbf{z}$ & Abund & Formula & Ion \\
\hline 323.1535 & 1 & 94497.52 & C23H18N2 & $(\mathrm{M}+\mathrm{H})+$ \\
\hline 324.1571 & 1 & 23877.73 & C23H18N2 & $(\mathrm{M}+\mathrm{H})+$ \\
\hline 325.1605 & 1 & 2688.8 & C23H18N2 & $(\mathrm{M}+\mathrm{H})+$ \\
\hline 326.1592 & 1 & 517.68 & C23H18N2 & $(\mathrm{M}+\mathrm{H})+$ \\
\hline 345.1344 & 1 & 746.88 & C23H18N2 & $(\mathrm{M}+\mathrm{Na})+$ \\
\hline 346.1376 & 1 & 272.17 & C23H18N2 & $(\mathrm{M}+\mathrm{Na})+$ \\
\hline
\end{tabular}




\section{Photophysical properties of 4a-d and 3a-d}
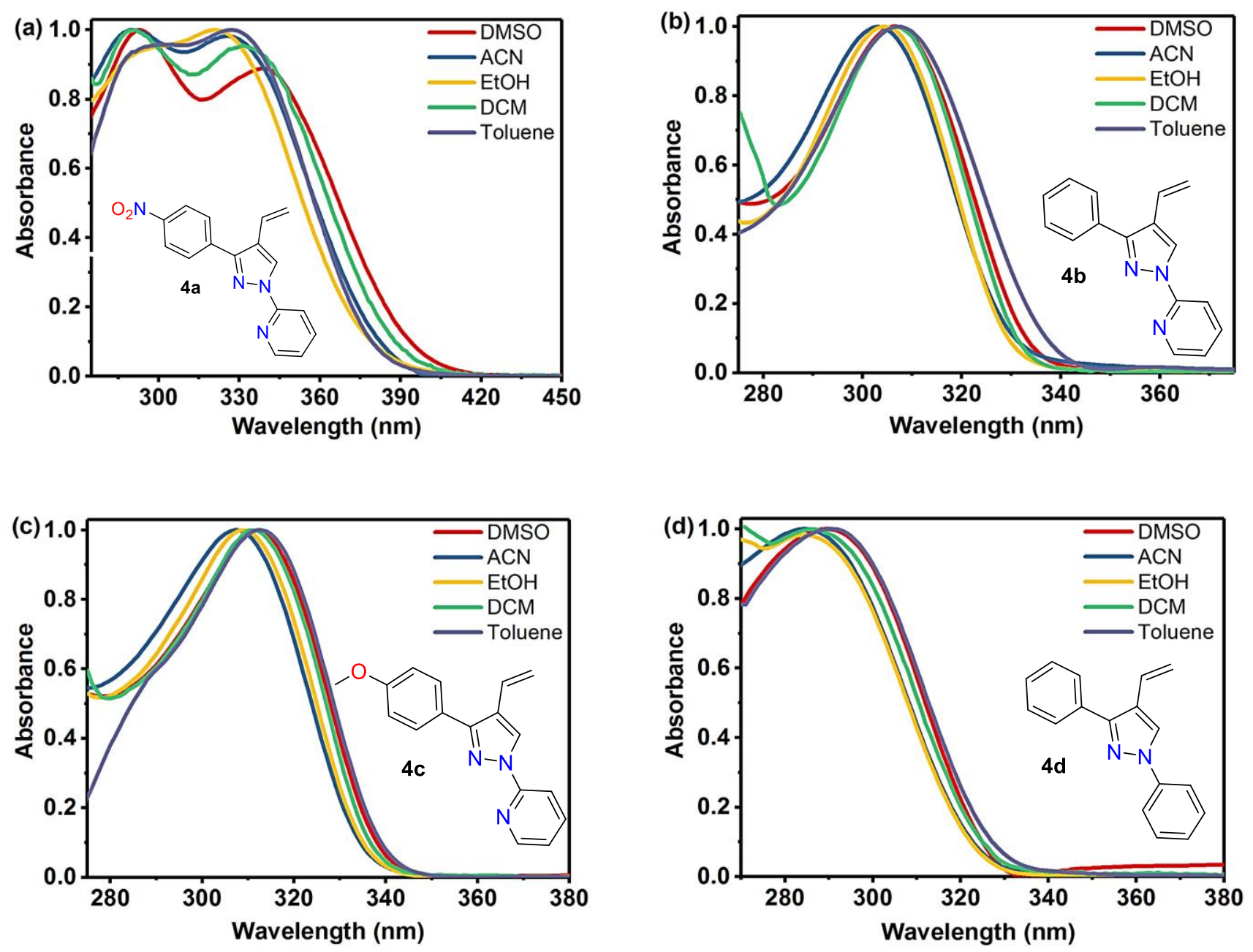

Figure S6. Normalized UV-vis spectra of (a) $\mathbf{4 a}$, (b) $\mathbf{4 b}$, (c) $\mathbf{4 c}$, and (d) $\mathbf{4 d}$ in various solvents $\left(1.0 \times 10^{-5} \mathbf{M}\right.$, $\left.20{ }^{\circ} \mathrm{C}\right)$. 

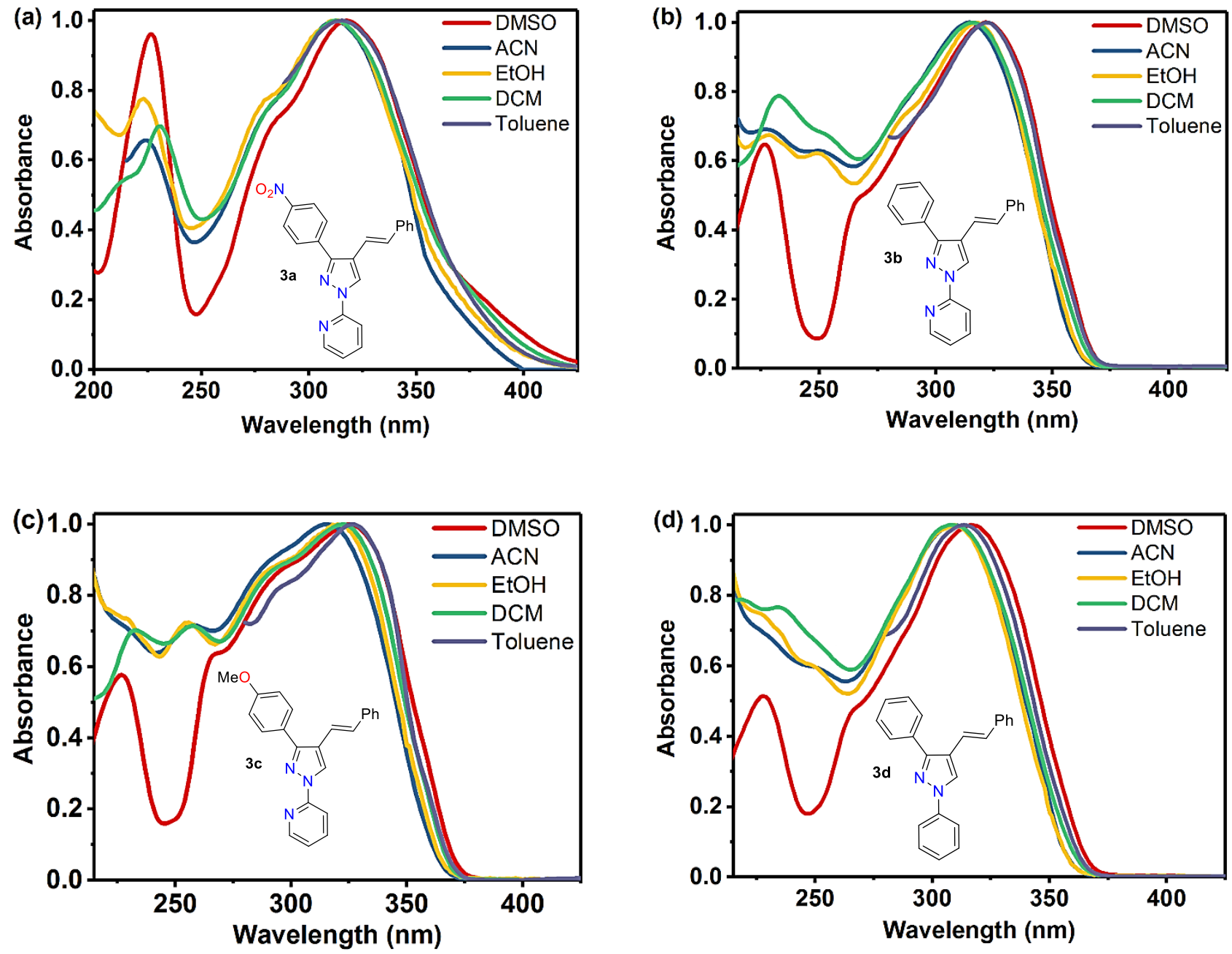

(e)

(f)
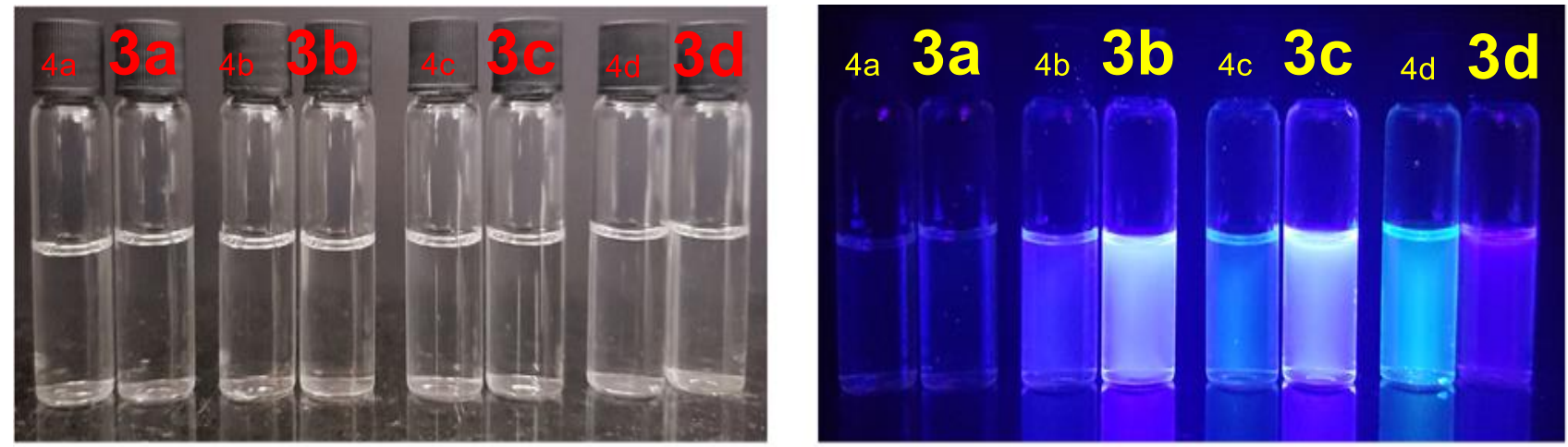

Figure S7. Normalized UV-vis spectra $\left(1.0 \times 10^{-5} \mathrm{M}\right)$ of (a) 3a, (b) $\mathbf{3 b}$, (c) 3c, and (d) 3d in different solvents at $20{ }^{\circ} \mathrm{C}$. The comparative photographs were taken using $2.0 \times 10^{-5} \mathrm{M}$ solutions of each compound in EtOH under (e) natural light and (f) using a hand-held UV lamp ( $\left.\lambda_{\mathrm{ex}}=365 \mathrm{~nm}\right)$. 

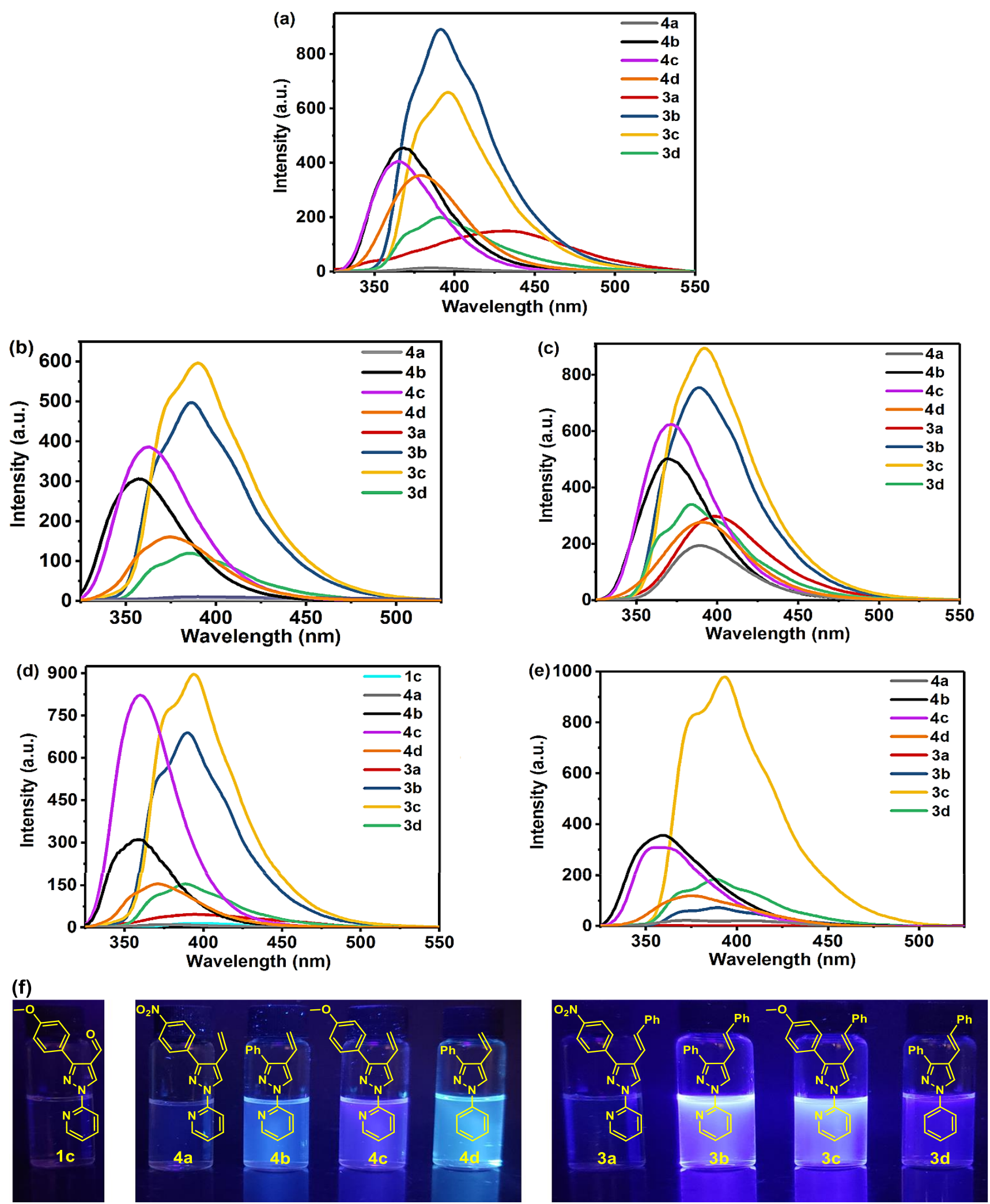

Figure S8. Fluorescence spectra of 1c, 4a-d, and 3a-d excited at the maximum absorption wavelength in different solvents $\left(1.0 \times 10^{-5} \mathrm{M}\right)$ at $20{ }^{\circ} \mathrm{C}$ : (a) DMSO, (b) ACN, (c) EtOH, (d) DCM, and (e) toluene. (f) Photographs were taken using $2.0 \times 10^{-5} \mathrm{M}$ solutions of each compound under an UV lamp $\left(\lambda_{\mathrm{ex}}=365 \mathrm{~nm}\right)$. 
Table S1. Photophysical properties of compounds 3a-d and 5a-d

\begin{tabular}{|c|c|c|c|c|c|c|}
\hline Compound & Solvent & $\lambda_{\mathrm{ab}}(\mathrm{nm})^{a}$ & $\varepsilon\left(\mathrm{L} \mathrm{mol}^{-1} \mathrm{~cm}^{-1}\right)$ & $\lambda_{\mathrm{em}}(\mathrm{nm})$ & Stokes shift (nm) & $\phi_{\mathrm{F}}^{b}$ \\
\hline \multirow{5}{*}{$\mathbf{4 a}^{c}$} & DMSO & 290 & 13830 & 360 & 70 & 0.01813 \\
\hline & $\mathrm{ACN}$ & 290 & 16175 & 357 & 67 & 0.00147 \\
\hline & $\mathrm{EtOH}$ & 320 & 16704 & 365 & 45 & 0.01542 \\
\hline & DCM & 290 & 15990 & 357 & 67 & 0.02426 \\
\hline & Toluene & 327 & 14520 & 348 & 21 & 0.06143 \\
\hline \multirow{5}{*}{$4 b^{c}$} & DMSO & 306 & 21137 & 357 & 51 & 0.47185 \\
\hline & $\mathrm{ACN}$ & 303 & 19157 & 350 & 47 & 0.27450 \\
\hline & $\mathrm{EtOH}$ & 305 & 15408 & 360 & 55 & 0.62930 \\
\hline & DCM & 307 & 19932 & 355 & 48 & 0.29719 \\
\hline & Toluene & 308 & 16606 & 352 & 44 & 0.49195 \\
\hline \multirow{5}{*}{$4 c^{c}$} & DMSO & 312 & 19734 & 365 & 53 & 0.48645 \\
\hline & $\mathrm{ACN}$ & 307 & 19857 & 361 & 54 & 0.37423 \\
\hline & $\mathrm{EtOH}$ & 309 & 20726 & 366 & 57 & 0.66116 \\
\hline & DCM & 307 & 19932 & 360 & 53 & 0.43508 \\
\hline & Toluene & 313 & 19476 & 352 & 39 & 0.37061 \\
\hline \multirow{5}{*}{$\mathbf{4 d}^{c}$} & DMSO & 289 & 12638 & 354 & 65 & 0.36723 \\
\hline & $\mathrm{ACN}$ & 284 & 17135 & 350 & 66 & 0.09385 \\
\hline & $\mathrm{EtOH}$ & 266 & 24219 & 360 & 94 & 0.14131 \\
\hline & DCM & 286 & 15671 & 351 & 65 & 0.12113 \\
\hline & Toluene & 290 & 15941 & 351 & 61 & 0.09845 \\
\hline \multirow{5}{*}{$3 \mathbf{a}^{d}$} & DMSO & 318 & 27413 & 433 & 115 & 0.04346 \\
\hline & $\mathrm{ACN}$ & 312 & 21931 & 390 & 78 & 0.00297 \\
\hline & $\mathrm{EtOH}$ & 312 & 20860 & 399 & 87 & 0.04370 \\
\hline & DCM & 313 & 27413 & 394 & 81 & 0.00902 \\
\hline & Toluene & 315 & 38416 & 325 & 10 & 0.00043 \\
\hline \multirow{5}{*}{$3 \mathbf{b}^{d}$} & DMSO & 322 & 32132 & 391 & 69 & 0.37910 \\
\hline & $\mathrm{ACN}$ & 314 & 25347 & 386 & 72 & 0.21493 \\
\hline & $\mathrm{EtOH}^{e}$ & 318 & 35624 & 389 & 71 & 0.45498 \\
\hline & DCM & 316 & 39205 & 389 & 73 & 0.24526 \\
\hline & Toluene & 322 & 30638 & 389 & 67 & 0.03779 \\
\hline \multirow{5}{*}{$3 c^{d}$} & DMSO & 322 & 23428 & 396 & 74 & 0.32800 \\
\hline & $\mathrm{ACN}$ & 315 & 36482 & 390 & 75 & 0.20067 \\
\hline & $\mathrm{EtOH}$ & 320 & 21183 & 392 & 72 & 0.42683 \\
\hline & DCM & 322 & 30754 & 394 & 72 & 0.34530 \\
\hline & Toluene & 326 & 31639 & 393 & 67 & 0.51172 \\
\hline \multirow{5}{*}{$3 \mathbf{d}^{d}$} & DMSO & 317 & 79218 & 390 & 73 & 0.22292 \\
\hline & $\mathrm{ACN}$ & 309 & 28969 & 385 & 76 & 0.04686 \\
\hline & $\mathrm{EtOH}$ & 310 & 31307 & 384 & 74 & 0.12645 \\
\hline & DCM & 308 & 27546 & 389 & 81 & 0.06920 \\
\hline & Toluene & 314 & 34620 & 388 & 74 & 0.08345 \\
\hline
\end{tabular}

${ }^{a} 1.0 \times 10^{-5} \mathrm{M} .{ }^{b}$ Relative quamtun yields were taken using quinine sulfate as a reference $\left(\phi_{\mathrm{F}}=0.59\right.$ in $0.15 \mathrm{M}$ $\left.\mathrm{HClO}_{4}\right)$. Excited at the maximum absorption wavelength. ${ }^{c} 1.0 \times 10^{-5} \mathrm{M} \cdot{ }^{d} 5.0 \times 10^{-5} \mathrm{M} .{ }^{e} 2.5 \times 10^{-5} \mathrm{M}$. 


\section{Chemosensor design 3b}
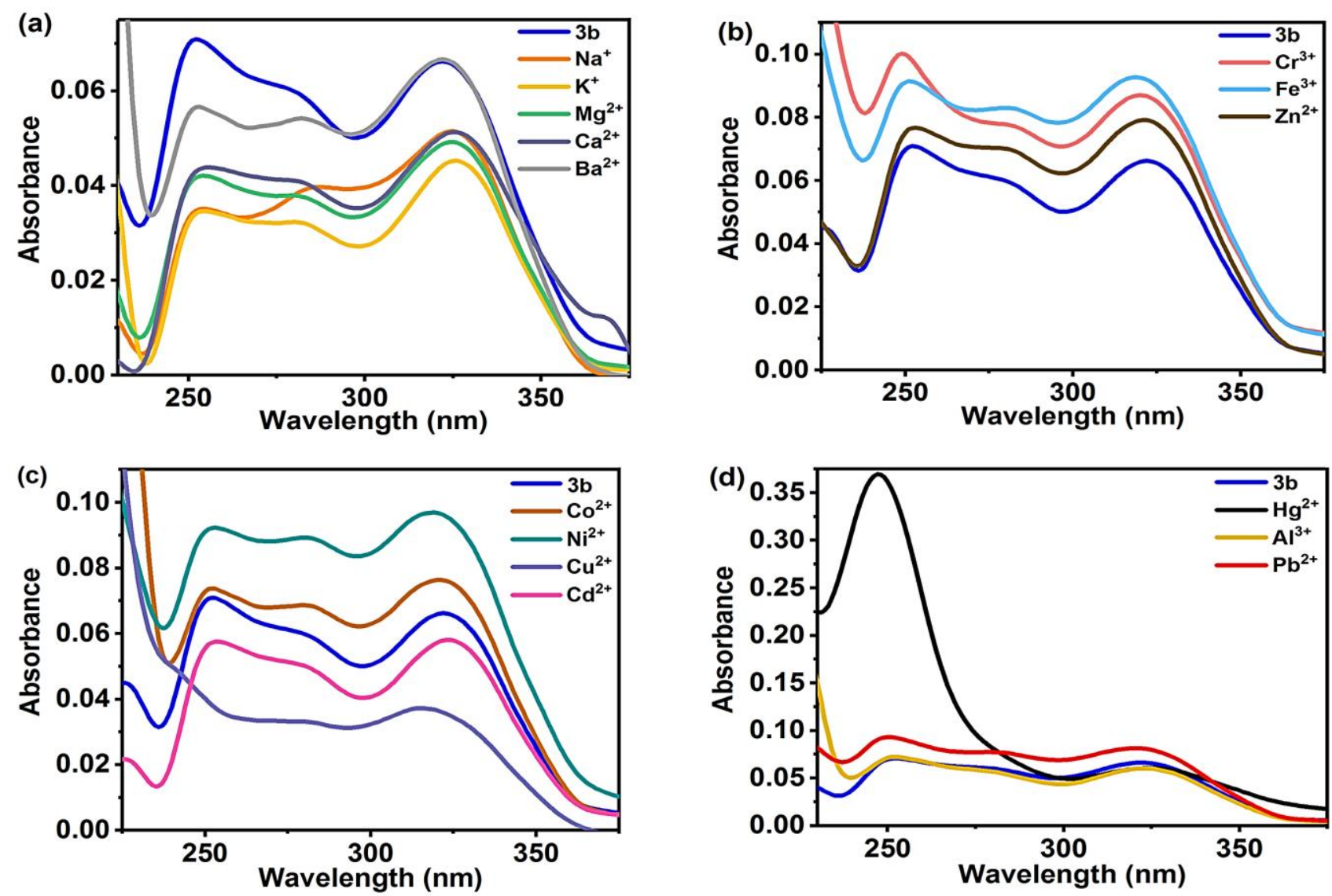

Figure S9. UV-vis spectra of $\mathbf{3 b}\left(4.0 \times 10^{-6} \mathrm{M}\right.$ in EtOH: $\left.\mathrm{H}_{2} \mathrm{O}, 9: 1 \mathrm{v} / \mathrm{v}\right)$ at $20{ }^{\circ} \mathrm{C}$ after the addition of an excess of different metal ions $\left(4.0 \times 10^{-4}, 100\right.$ equiv of each metal ion).

(a)

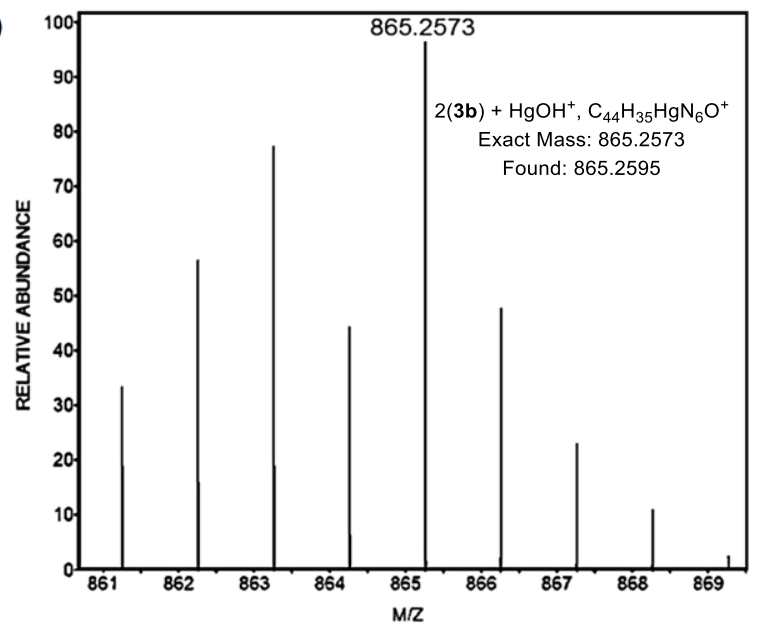

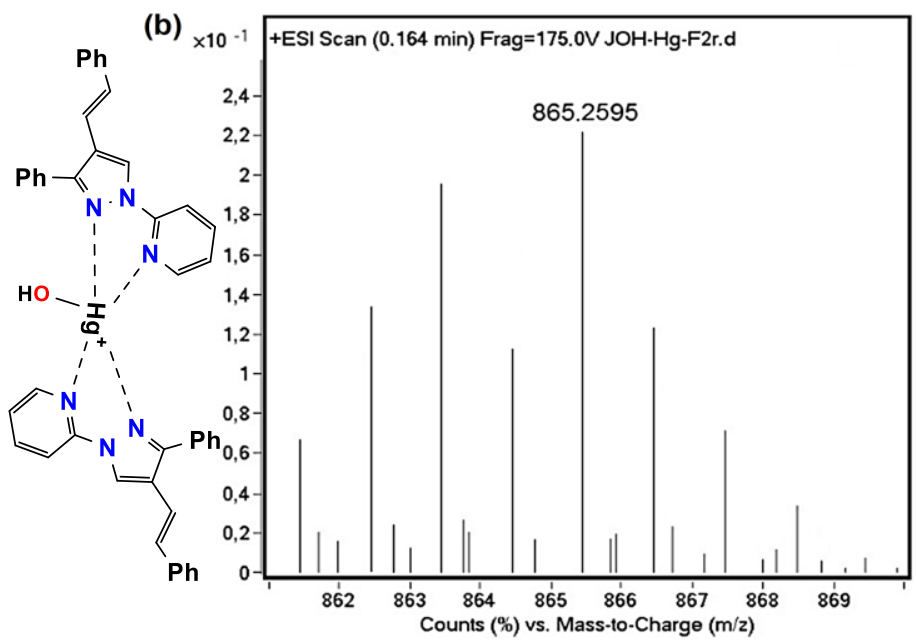

Figure S10. (a) Isotopic distribution calculated for the $[2 \cdot 3 \mathbf{b}+\mathrm{HgOH}]^{+}$complex. (b) HRMS (ESI+) spectrum for the $2: 1[2 \cdot 3 \mathbf{b}+\mathrm{HgOH}]^{+}$complex in $\mathrm{MeOH}: \mathrm{H}_{2} \mathrm{O}(1: 1 \mathrm{v} / \mathrm{v})$. 